\title{
THE STRUCTURE OF MEAN EQUICONTINUOUS GROUP ACTIONS*
}

\author{
BY \\ Gabriel Fuhrmann \\ Department of Mathematical Sciences, Durham University \\ Mathematical Sciences $\&$ Computer Science Building, Upper Mountjoy Campus \\ Stockton Road, Durham DH1 3LE, UK \\ e-mail: gabriel.fuhrmann@durham.ac.uk
}

AND

MAIK GRÖGER

Faculty of Mathematics, University of Vienna

Oskar-Morgenstern-Platz 1, 1090 Vienna, Austria

and

Faculty of Mathematics and Computer Science, Jagiellonian University in Krakow ul. Eojasiewicza 6, 30-348 Kraków, Poland e-mail: maik.groeger@im.uj.edu.pl

\author{
AND \\ DANIEL LENZ \\ Department of Mathematics, University of Jena \\ Ernst-Abbe-Platz 2, 07743 Jena, Germany \\ e-mail:daniel.lenz@uni-jena.de
}

\footnotetext{
* (C) The authors 2018. This article is published with open access at link.springer.com. Open Access This article is distributed under the terms of the Creative Commons Attribution 4.0 International License (https://creativecommons.org/licenses/by/4.0/), which permits unrestricted use, distribution and reproduction in any medium, provided the appropriate credit is given to the original authors and the source, and a link is provided to the Creative Commons license, indicating if changes were made.
} Received September 15, 2020 and in revised form December 10, 2020 


\section{ABSTRACT}

We study mean equicontinuous actions of locally compact $\sigma$-compact amenable groups on compact metric spaces. In this setting, we establish the equivalence of mean equicontinuity and topo-isomorphy to the maximal equicontinuous factor and provide a characterization of mean equicontinuity of an action via properties of its product. This characterization enables us to show the equivalence of mean equicontinuity and the weaker notion of Besicovitch-mean equicontinuity in fairly high generality, including actions of abelian groups as well as minimal actions of general groups. In the minimal case, we further conclude that mean equicontinuity is equivalent to discrete spectrum with continuous eigenfunctions. Applications of our results yield a new class of non-abelian mean equicontinuous examples as well as a characterization of those extensions of mean equicontinuous actions which are still mean equicontinuous.

\section{Introduction}

Isometric actions on compact metric spaces constitute fundamental objects of study in the field of dynamical systems. In fact, despite possessing structurally simple dynamics, they relate to deep problems of general mathematical interest. Already rigid rotations on the circle have close connections to continued fraction expansions (see, for example, [Ser85]), the rich theory of discrepancy of sequences (see, e.g., [DT97] and references therein), or the Three Distance Problem and its versatile generalizations (see, for instance, [AB98]), to name but a few. With their dynamical simplicity on the one hand and the relevance of such problems on the other hand, it is natural to take actions by isometries as a point of departure in the endeavor to understand topological dynamical systems in general.

Actually, a substantial part of the abstract theory of topological dynamics can be understood as dealing with the following issue: given a general action which is not isometric, how close is this action to an isometric one? An essential tool in answering this question is the so-called maximal equicontinuous factor (or, topologically equivalent, the maximal isometric factor) of a given action. Now, with this canonical factor at hand, we may restate the above question in the following way: what is the regularity of the corresponding factor map?

Of course, various regularity features can be (and have been) considered. On the topological side, it is natural to investigate the existence of points where this factor map is one-to-one and this leads to the notion of almost automorphic 
actions [Vee65]. Once an invariant measure $\mu$ is given, one can also ask for injectivity of the factor map for almost all points with respect to $\mu$ and in many contexts this is referred to as regularity of the system.

With a more measure-theoretical flavor, we may study factor maps that establish a measure isomorphy with respect to all invariant measures and their push-forward on the maximal equicontinuous factor. This is the starting point of the current article and we present a comprehensive treatment of actions which allow for such factor maps. Our first main result gives a characterization of these actions in terms of a weakening of isometry known as mean equicontinuity (Theorem 1.1). Our subsequent results then unfold the notion of mean equicontinuity in terms of product systems (Theorem 1.2) and provide a spectral characterization of mean equicontinuity (Theorem 1.4) for minimal actions. A priori, the concept of mean equicontinuity comes in two variants, one known as Weyl-mean equicontinuity and the other as Besicovitch-mean equicontinuity. Along the way, we derive sufficient conditions for these two notions to agree (Theorem 1.3).

The concepts of Weyl- and Besicovitch-mean equicontinuity were introduced in [LTY15] for integer actions. In fact, in this case the notion of Besicovitchmean equicontinuity is immediately seen to be equivalent to the concept of mean Lyapunov-stability which was already introduced in 1951 by Fomin [Fom51] in the context of $\mathbb{Z}$-actions with discrete spectrum. Later, a first systematic treatment was carried out by Auslander [Aus59].

Our results tie in with various recent streaks of investigations: for $\mathbb{Z}$-actions, there is the fundamental work of Downarowicz and Glasner on mean equicontinuity [DG16], providing a detailed study in the minimal case. Our results generalize these results from the group of integers to general locally compact $\sigma$-compact amenable groups. In the main structural characterization given in Theorem 1.1, we can also completely remove the minimality condition. Further, in our treatment of the relation between Weyl- and Besicovitch-mean equicontinuity, we can remove the minimality condition in many cases as well and thereby generalize [QZ20] which treats the case of general (that is, not necessarily minimal) $\mathbb{Z}$-actions.

Concerning abelian groups, mean equicontinuity and its relation to the spectral theory of dynamical systems (in particular, to discrete spectrum) has been studied by various groups [GR17, Len19, GRM19]. Indeed, these works feature weaker versions of mean equicontinuity in order to characterize discrete 
spectrum. So, the restriction of our spectral result to the abelian case, given in Corollary 1.6, can be seen as a natural complement to these works. More specifically, our spectral characterization shows -in the minimal case- that mean equicontinuity is equivalent to unique ergodicity and discrete spectrum together with the continuity of eigenfunctions (see also [DG16] for the case of $\mathbb{Z}$-actions).

Discrete spectrum is particularly relevant in the context of aperiodic order. This field has attracted substantial attention in the last decades due to the discovery of substances - later called quasicrystals - featuring this type of order (see the recent survey collection [KLS15] and the monograph [BG13] for background and further details). A basic quantity in the study of aperiodic order is the diffraction measure of an aperiodic configuration and a key task is to understand when the diffraction measure is a pure point measure. Due to a collective effort over the last twenty years, this turns out to be equivalent to discrete spectrum of an associated dynamical systems; see, for instance, [BL17] for a recent survey.

There is no axiomatic framework for aperiodic order (yet). However, typical systems studied in the context of aperiodic order have further regularity properties such as minimality and unique ergodicity. As discussed below (see Remark 6.4), one may argue that our spectral characterization shows that mean equicontinuous systems are the "right" systems to model minimal systems with aperiodic order.

1.1. Basic notation And Definitions. We call a triple $(X, G, \alpha)$ a (topological) dynamical system if $X$ is a compact metric space (endowed with a metric $d), G$ is a topological group and $\alpha$ is a continuous action of $G$ on $X$ by homeomorphisms. Here, continuity of $\alpha$ is understood as continuity of the map

$$
G \times X \ni(g, x) \mapsto \alpha(g)(x) \in X .
$$

Most of the time, we will keep the action $\alpha$ implicit and simply refer to $(X, G)$ as a dynamical system. In a similar fashion, we mostly write $g x$ instead of $\alpha(g)(x)$ $(g \in G, x \in X)$. For $\mathbb{Z}$-actions, which are uniquely determined by $f:=\alpha(1)$, we also refer by $(X, f)$ to the dynamical system $(X, \mathbb{Z}, \alpha)$.

A dynamical system $(Y, G)$ is a (topological) factor of another dynamical system $(X, G)$ if there exists a continuous surjection $h: X \longrightarrow Y$ with

$$
h(g x)=g h(x)
$$


for all $g \in G$ and $x \in X$. In this case, $h$ is called a factor map and $(X, G)$ is referred to as a (topological) extension of $(Y, G)$. If $h$ is further injective (and hence, a homeomorphism), we say $(X, G)$ and $(Y, G)$ are conjugate and call $h$ a conjugacy.

We say $(X, G)$ is transitive if there is $x \in X$ whose orbit $G x$ is dense. In this case, also the point $x$ is called transitive. We say $(X, G)$ is minimal if every $x \in X$ is transitive. A set $A \subseteq X$ is invariant under $G$ (or $G$-invariant) if $g A=A$ for all $g \in G$. We call a non-empty, closed and $G$-invariant set $A \subseteq X$ transitive if $(A, G)$ is transitive; we call such a set minimal if $(A, G)$ is minimal. It is a well-known consequence of Zorn's Lemma that every dynamical system $(X, G)$ has a minimal set $A \subseteq X$. Clearly, distinct minimal sets are disjoint. Observe that invariance, transitivity and minimality are preserved under factor maps.

A system $(X, G)$ is equicontinuous if for every $\varepsilon>0$ there exists $\delta_{\varepsilon}>0$ such that $d(x, y)<\delta_{\varepsilon}$ implies $d(g x, g y)<\varepsilon$ for all $g \in G$. If $\delta_{\varepsilon}$ can be chosen to equal $\varepsilon$, then $(X, G)$ is called isometric. Observe that if $(X, G)$ is equicontinuous, then

$$
\hat{d}(x, y)=\sup _{g \in G} d(g x, g y)
$$

defines a metric that induces the same topology on $X$ as $d$. Clearly,

$$
\hat{d}(g x, g y)=\hat{d}(x, y)
$$

for all $g \in G$ and $x, y \in X$ which implies that we can use the terms equicontinuous system and isometric system synonymously.

It is well known that every topological dynamical system $(X, G)$ has a unique (up to conjugacy) maximal equicontinuous factor (MEF), denoted by $(\mathbb{T}, G)$. That is, $(\mathbb{T}, G)$ is an equicontinuous factor of $(X, G)$ and moreover an extension of every other equicontinuous factor of $(X, G)$. For our considerations, the following simple consequence of the defining property of $(\mathbb{T}, G)$ will be sufficient: If ( $\left.\mathbb{T}^{\prime}, G\right)$ is an equicontinuous factor of $(X, G)$ with factor map $\pi^{\prime}$ such that $\pi^{\prime}(x)=\pi^{\prime}(y)$ implies

$$
\inf _{g \in G} d(g x, g y)=0,
$$

then $\left(\mathbb{T}^{\prime}, G\right)$ is conjugate to $(\mathbb{T}, G)$. For a detailed discussion of the above facts, we refer to [Aus88]. 
Throughout this work, we consider $G$ to be a locally compact $\sigma$-compact amenable group. Recall that a locally compact $\sigma$-compact group $G$ is called amenable if there exists a sequence $\left(F_{n}\right)_{n \in \mathbb{N}}$, called a (left) Følner sequence, of non-empty compact sets in $G$ of positive Haar measure such that

$$
\lim _{n \rightarrow \infty} \frac{m\left(g F_{n} \triangle F_{n}\right)}{m\left(F_{n}\right)}=0 \quad \text { for all } g \in G,
$$

where $\triangle$ denotes the symmetric difference and $m$ is a (left) Haar measure of $G$ (we may synonymously write $|F|$ for the Haar measure $m(F)$ of a measurable set $F \subseteq G)$.

Since $G$ acts by homeomorphisms, for each $g \in G$ the map $g: X \ni x \mapsto g x$ is Borel bi-measurable. We call a Borel probability measure $\mu$ on $X$ invariant under $G$ (or $G$-invariant) if $\mu(A)=\mu(g A)$ for every Borel measurable subset $A \subseteq X$ and $g \in G$. We say a $G$-invariant measure $\mu$ is ergodic if all Borel sets $A$ with $\mu(A \triangle g A)=0(g \in G)$ verify $\mu(A)=0$ or $\mu(A)=1$. It is well known that the amenability of $G$ ensures the existence of a $G$-invariant measure for $(X, G)$. Further, the set of invariant measures is convex and an invariant measure is ergodic if and only if it is an extremal point of the set of invariant measures. In particular, if $(X, G)$ has a unique invariant measure, this measure is necessarily ergodic and $(X, G)$ is referred to as uniquely ergodic. Finally, we call a closed invariant set $A \subseteq X$ uniquely ergodic if $(A, G)$ is uniquely ergodic. For further information of measure-theoretic properties of dynamical systems, see also [EW11].

1.2. Main Results. Given a dynamical system $(X, G)$ and a Følner sequence $\mathcal{F}=\left(F_{n}\right)_{n \in \mathbb{N}}$, we call $(X, G)$ Besicovitch- $\mathcal{F}$-mean equicontinuous or just $\mathcal{F}$-mean equicontinuous if for all $\varepsilon>0$ there exists $\delta_{\varepsilon}>0$ such that

$$
D_{\mathcal{F}}(x, y):=\varlimsup_{n \rightarrow \infty} \frac{1}{\left|F_{n}\right|} \int_{F_{n}} d(t x, t y) d m(t)<\varepsilon,
$$

for all $x, y \in X$ with $d(x, y)<\delta_{\varepsilon}$. The dependence on the $\mathrm{F} \varnothing$ lner sequence immediately motivates the next definition which will also be the integral notion in this article. We say $(X, G)$ is Weyl-mean equicontinuous or just mean equicontinuous if for all $\varepsilon>0$ there is $\delta_{\varepsilon}>0$ such that for all $x, y \in X$ with $d(x, y)<\delta_{\varepsilon}$ we have

$$
D(x, y):=\sup \left\{D_{\mathcal{F}}(x, y) \mid \mathcal{F} \text { is a } \mathrm{F} \varnothing \text { lner sequence }\right\}<\varepsilon .
$$


Before we can proceed, a few comments are in order. First, note that $D_{\mathcal{F}}$ and $D$ are pseudometrics. Moreover, as is not hard to see, $D$ is $G$-invariant, that is, $D(g x, g y)=D(x, y)$ for all $x, y \in X$ and $g \in G$ (for the convenience of the reader, we provide a proof of this fact, see Proposition 3.12). Indeed, if $G$ is abelian, it is immediately seen that (1) already defines a $G$-invariant pseudometric (simply for algebraic reasons) which simplifies many proofs for abelian $G$. In the non-abelian situation, this does not hold anymore in general. Yet, it turns out that under fairly general assumptions on $(X, G)$ it actually is true if $(X, G)$ is mean equicontinuous (see Theorem 1.3). It is an interesting observation that in this case, however, the reason behind the invariance of $D_{\mathcal{F}}$ is not so much algebraic but ergodic in nature (see Section 5).

In the following main structural result, we will see that mean equicontinuity of a system $(X, G)$ is intimately linked to a regularity property of the topological factor map $\pi: X \rightarrow \mathbb{T}$ onto its maximal equicontinuous factor $(\mathbb{T}, G)$. For the definition of this regularity property, we need to introduce the following notion. Two probability spaces $\left(X, \mathcal{B}_{X}, \mu\right)$ and $\left(Y, \mathcal{B}_{Y}, \nu\right)$ are called isomorphic $(\bmod 0)$ if there are measurable sets $M \subseteq X$ and $N \subseteq Y$ with

$$
\mu(M)=\nu(N)=1
$$

and a bi-measurable bijection $h^{\prime}: M \rightarrow N$ which is measure preserving, that is,

$$
\mu\left(h^{\prime-1}(A)\right)=\nu(A)
$$

for all measurable $A \subseteq N$. In this case, we call $h^{\prime}$ an isomorphism $(\bmod 0)$ with respect to $\mu$ and $\nu$. We also refer to an everywhere defined measurable map $h: X \rightarrow Y$ as an isomorphism (mod 0) with respect to $\mu$ and $\nu$ if $h(x)=h^{\prime}(x)$ with $x \in M$ for some $h^{\prime}$ and $M$ as above.

Suppose now that $(X, G)$ is a topological extension of $(Y, G)$ via a factor map $h: X \rightarrow Y$ and let $\mu$ be a $G$-invariant measure on $X$. We say $(X, G)$ is a topo-isomorphic extension of $(Y, G)$ with respect to $\mu$ if $h$ is also an isomorphism with respect to $\mu$ and $h(\mu)$ where $h(\mu)$ denotes the push-forward of $\mu$. In this case, we call $h$ a topo-isomorphy with respect to $\mu$. In case that no measure is specified, $(X, G)$ is called a topo-isomorphic extension of $(Y, G)$ and $h$ a topo-isomorphy if $h: X \rightarrow Y$ is a topo-isomorphy with respect to every $G$-invariant measure $\mu$ on $X$. Observe that the push-forward of an invariant measure $\mu$ under a topo-isomorphy is ergodic if and only if $\mu$ is ergodic. 
Theorem 1.1 (Mean equicontinuity and topo-isomorphy): The topological $d y$ namical system $(X, G)$ is (Weyl-) mean equicontinuous if and only if it is a topo-isomorphic extension of its maximal equicontinuous factor $(\mathbb{T}, G)$.

Let us point out that the proof of this theorem also shows that the maximal equicontinuous factor of a mean equicontinuous system is in a natural sense the quotient of $X$ by the pseudometric $D$ (see the corresponding discussion in Section 3.3).

The concept of topo-isomorphy is at the interface of topological and measuretheoretical aspects of dynamical systems. This kind of "hybrid" notion was also recently studied by Downarowicz and Glasner in [DG16] where a similar statement to the above is proven for minimal dynamical systems with $G=\mathbb{Z}$. We would like to mention that the direction from topo-isomorphy to mean equicontinuity (Theorem 3.7) is proven in a completely different way than in [DG16], while the proof that mean equicontinuity implies topo-isomorphy is close to the ones of [LTY15, Theorem 3.8] and [DG16, Proposition 2.5]; see Section 3 .

It is very worth noting that Theorem 1.1 is by far not only of abstract importance but actually offers a direct way to establish the mean equicontinuity of many well-known minimal group actions. To emphasize this, we briefly present a (non-exhaustive) list of minimal group actions where mean equicontinuity can always be derived by using the structural characterization provided in Theorem 1.1. Starting with $\mathbb{Z}$-actions, two very common example classes which are well known to be mean equicontinuous are Sturmian subshifts and regular Toeplitz subshifts; see, for instance, [Fog02, Ků03, Dow05] for further information and references. Non-symbolic examples can be found in the class of so-called Auslander systems (see [Aus88] and [HJ97]).

Before we go beyond $\mathbb{Z}$-actions, we want to stress that minimal mean equicontinuous systems are always uniquely ergodic; see Corollary 1.5 (iii). For $G=\mathbb{Z}$, this is a classical observation which is due to Oxtoby [Oxt52]; see also [Aus59]. To present the reader a non-minimal and moreover, intrinsically non-uniquely ergodic system, we provide a symbolic $\mathbb{Z}$-action which has infinitely many ergodic measures in Example 5.11.

Concerning actions by more general groups, Theorem 1.1 also constitutes a basis for providing a novel and straightforward construction method for a class of non-abelian minimal mean equicontinuous systems (outlined in Subsection 7.1). 
Moreover, we can continue the list of examples from above: higher-dimensional subshifts, i.e., $\mathbb{Z}^{n}$-actions, which are mean equicontinuous can for instance be obtained from regular Toeplitz arrays; see [Cor06]. Furthermore, the theory of quasicrystals contains many natural examples of mean equicontinuous $\mathbb{R}^{n}$ actions, like the $\mathbb{R}^{2}$-actions obtained from Penrose tilings [Rob96] or the chair tiling [Rob99]. For more information concerning tilings and Delone sets in $\mathbb{R}^{n}$, see [BG13]. It is also possible to consider Delone sets (and canonical actions induced by them) in more general groups than $\mathbb{R}^{n}$. Especially, so-called regular model sets immediately yield mean equicontinuous group actions; see [Sch99].

Moreover, as recently shown in [GL20] and motivated by [Vor12], there exists a rich class of mean equicontinuous systems in the context of actions of self-similar groups on spherically homogeneous trees. One can associate canonical extensions to this kind of actions and in [GL20] natural conditions are established which ensure that these extensions act mean equicontinuously. In particular, all actions on $d$-ary trees of groups generated by bounded automata fulfil these conditions and many well-known groups belong to this class, including the Basilica and Grigorchuk group, as well as iterated monodromy groups associated to quadratic post-critically finite polynomials.

Finally, we would like to mention that according to [Gla18, Corollary 5.4 (2)], minimal tame systems are always topo-isomorphic extensions of their maximal equicontinuous factor if the corresponding acting group is amenable (see also the short discussion at the end of Section 7.3). Systems belonging to this family are Sturmian-like $\mathbb{Z}^{n}$-actions [GM18a] or tame generalized Toeplitz shifts [FK20] (see also [LS18] for not necessarily tame but still mean equicontinuous examples). In fact, in [FK20] it is shown that every countable maximally almost periodic amenable group allows for effective mean equicontinuous minimal actions which are not equicontinuous (see also Section 7.3).

Our next main result gives a characterization of mean equicontinuity of a system in terms of its product system (see Section 4 for details). It is to be seen as a generalization of [LTY15, Theorem 3.3] and thereby well in line with a plethora of results on characterizing properties of a dynamical system via properties of its product. We need the following notion: a system $(X, G)$ is pointwise uniquely ergodic if the orbit closure $\overline{G x}$ of every point $x \in X$ is uniquely ergodic. For such systems we denote by $\mu_{x}$ the unique ergodic measure supported on the orbit closure of $x \in X$. 
Theorem 1.2 (Mean equicontinuity and the product system): The system $(X, G)$ is mean equicontinuous if and only if

- the product system $(X \times X, G)$ is pointwise uniquely ergodic,

- and the map $(x, y) \mapsto \mu_{(x, y)}$ is continuous (with respect to the weak*topology).

As the metric $d$ is continuous on $X \times X$, the previous theorem together with the standard result on the existence of averages of continuous functions for uniquely ergodic dynamical systems implies for mean equicontinuous systems that the limsup in (1) is actually a limit and does not depend on the chosen Følner sequence, whence, in particular, it follows that $D=D_{\mathcal{F}}$ for any left Følner sequence $\mathcal{F}$ (see also Section 4 for a related discussion).

Moreover, the previous result allows us to derive the following theorem on the independence of Følner sequences.

Theorem 1.3 (Mean equicontinuity and $\mathcal{F}$-mean equicontinuity): Let $(X, G)$ be a dynamical system and assume that

- there is an invariant measure $\mu$ with full support, i.e., $\operatorname{supp}(\mu)=X$, or that

- the group $G$ is abelian.

Then $(X, G)$ is mean equicontinuous if and only if $(X, G)$ is $\mathcal{F}$-mean equicontinuous for some left Følner sequence $\mathcal{F}$.

Observe that if $(X, G)$ is minimal, the extra assumption of a measure with full support is evidently fulfilled. It is noteworthy that the extra effort needed to overcome the lack of commutativity in this work is most visible in the proof of the above statement. We would also like to remark that in the recent article [QZ20], a similar statement has independently (and by different means) been proven to hold if $G=\mathbb{Z}$. Under the assumption of a minimal $\mathbb{Z}$-action, it is known due to [DG16].

In the minimal case we can provide another characterization of mean equicontinuity. This is a characterization in terms of spectral theory, or more specifically, in terms of a decomposition of the space $L^{2}(X, \mu)$. The corresponding proof can be found in Section 6 .

Theorem 1.4 (Mean equicontinuity and spectral theory): Assume $(X, G)$ is minimal. Then $(X, G)$ is mean equicontinuous if and only if $(X, G)$ has a unique invariant measure $\mu$ and $L^{2}(X, \mu)$ can be written as an orthogonal sum of finitedimensional, $G$-invariant subspaces consisting of continuous functions. 
Now, for minimal systems we may combine all the previous theorems to obtain a slightly simplified list of equivalent characterizations of mean equicontinuity (note that the statements (i)-(iii) are a generalization of Theorem 2.1 in [DG16] which is treating $\mathbb{Z}$-actions).

Corollary 1.5: Let $(X, G)$ be a minimal system. Then the following are equivalent:

(i) $(X, G)$ is mean equicontinuous.

(ii) $(X, G)$ is $\mathcal{F}$-mean equicontinuous for some (left) Følner sequence $\mathcal{F}$.

(iii) $(X, G)$ is uniquely ergodic and topo-isomorphic to its $M E F$ with respect to its unique invariant measure $\mu$.

(iv) $(X \times X, G)$ is pointwise uniquely ergodic and $(x, y) \mapsto \mu_{(x, y)}$ is continuous.

(v) $(X, G)$ has a unique invariant measure $\mu$ and $L^{2}(X, \mu)$ can be written as an orthogonal sum of finite-dimensional, $G$-invariant subspaces consisting of continuous functions.

It is worth mentioning that the last point of the above statement reflects the close connection between discrete spectrum and properties of certain averaging pseudometrics; see also [GR17, $\mathrm{HLT}^{+} 21$, YZZ19] for recent investigations in this direction. Given the relevance of this connection, we finish the present section with a discussion of the spectral characterization of mean equicontinuity for abelian $G$. This case is particularly important due to its relevance for the study of aperiodic order. Let $\widehat{G}$ be the dual group of $G$, i.e., the group of all continuous group homomorphisms from $G$ to the unit circle and let $(X, G)$ be a dynamical system with an invariant probability measure $\mu$. Then $f \in L^{2}(X, \mu)$ with $f \neq 0$ is called an eigenfunction to the eigenvalue $\xi \in \widehat{G}$ if

$$
f(g \cdot)=\xi(g) f(\cdot)
$$

for all $g \in G$. Here, the equality is understood in the sense of $L^{2}$ functions. If such an $f$ is continuous with

$$
f(g x)=\xi(g) f(x),
$$

for all $x \in X$ and $g \in G$ it is called a continuous eigenfunction. The dynamical system $(X, G)$ with $G$-invariant measure $\mu$ is said to have discrete spectrum with continuous eigenfunctions if there exists an orthonormal basis for $L^{2}(X, \mu)$ of continuous eigenfunctions. 
Corollary 1.6: Let $G$ be abelian. A minimal system $(X, G)$ is mean equicontinuous if and only if it is uniquely ergodic and has discrete spectrum with continuous eigenfunctions.

For subshifts associated to non-periodic primitive substitutions, a classical result by Host [Hos86] states that all eigenvalues possess a continuous eigenfunction. Hence, the previous corollary implies that these subshifts are mean equicontinuous if and only if they have pure point spectrum (since they are always minimal). This yields, for instance, that the subshifts associated to the Fibonacci and Tribonacci substitution are mean equicontinuous. For more information, see for instance [Fog02] and [Que10]. Further, a generalization of Host's result to primitive tiling substitutions of $\mathbb{R}^{n}$ with finite local complexity can be found in [Sol07].

Acknowledgments. This project has received funding from the European Union's Horizon 2020 research and innovation program under the Marie Skłodowska-Curie grant agreement No 750865. Furthermore, MG acknowledges support by the DFG grants JA 1721/2-1 and GR 4899/1-1. Moreover, the authors would also like to thank Dominik Kwietniak for bringing the example depicted in Figure 1 to their attention.

\section{Some basic preliminaries on ergodic theory}

In this section, we discuss some definitions and statements of the ergodic theory of general actions by locally compact $\sigma$-compact amenable groups. To a large extent, we will be concerned with averages along $\mathrm{F} \varnothing l$ lner sequences where we pay special attention to an exposition which only requires a very fundamental set of tools. In particular, we will only make use of the Mean Ergodic Theorem in the following and avoid the more sophisticated Pointwise Ergodic Theorem by Lindenstrauss [Lin01].

In order to provide an alternative characterization of topo-isomorphic extensions, let us make the following classical measure-theoretic observation whose proof is provided for the convenience of the reader. 
Proposition 2.1: Suppose $X$ and $Y$ are compact metric spaces, $\mu$ is a Borel probability measure on $X$ and $h: X \rightarrow Y$ is measurable. Then, the operator

$$
U_{\mu}: L_{2}(Y, h(\mu)) \rightarrow L_{2}(X, \mu): f \mapsto f \circ h
$$

is unitary if and only if $h$ is an isomorphism (mod 0) with respect to $\mu$ and $h(\mu)$.

Proof. We only show that unitarity implies isomorphy (mod 0) the "if"-part is obvious.

First, we have to fix some notation. For a compact metric space $(Z, d)$ we denote by $\mathcal{B}(Z)$ the Borel $\sigma$-algebra and by $\tilde{\mathcal{B}}(Z)$ the associated measure algebra (see, for example, [Wal82] for the notion of measure algebras). Since $U_{\mu}: L_{2}(Y, h(\mu)) \rightarrow L_{2}(X, \mu)$ is unitary, we can define an invertible map $\tilde{\Phi}: \tilde{\mathcal{B}}(Y) \rightarrow \tilde{\mathcal{B}}(X)$ by setting $\tilde{\Phi}(\tilde{A})$ to be the equivalence class of $\Phi(A)$ in $\tilde{\mathcal{B}}(X)$, where

$$
U_{\mu}\left(\mathbf{1}_{A}\right)=\mathbf{1}_{\Phi(A)},
$$

for $A \in \tilde{A} \in \tilde{\mathcal{B}}(Y)$. Note that $\Phi(A)=h^{-1}(A)$ (this also proves the welldefinition of $\tilde{\Phi})$. One can check directly that $\tilde{\Phi}$ is a measure algebra isomorphism. Further, by [Wal82, Theorem 2.2] we conclude that there exist sets $M \subseteq X, N \subseteq Y$ with $\mu(M)=h(\mu)(N)=1$ and a Borel measurable invertible measure preserving map $\varphi: M \rightarrow N$ which induces $\tilde{\Phi}$, i.e.,

$$
\tilde{\Phi}(\tilde{A})=\left(\varphi^{-1}(A \cap N)\right)^{\sim}
$$

for all $A \in \mathcal{B}(Y)$, and coincides with $h$ on $M$. This proves the statement.

Recall that any locally compact group $G$ admits a left (right) Haar measure (defined uniquely up to a positive multiplicative constant) denoted by $m\left(m_{r}\right)$ which is left (right) invariant, that is, for all $\varphi \in L_{1}(G, m)$ and $g \in G$ we have

$$
\int \varphi(g s) d m(s)=\int \varphi(s) d m(s)
$$

$\left(\int \varphi(s g) d m_{r}(s)=\int \varphi(s) d m_{r}(s)\right)$, where $L_{1}(G, m)$ is the space of all Haar integrable functions on $G$. Note that from time to time we will also refer to the left/right Haar measure by using the notation $|\cdot|$ if there is no risk of ambiguity.

In the introduction we have already encountered the notion of a left Følner sequence $\left(F_{n}\right)_{n \in \mathbb{N}}$ in $G$ consisting of non-empty compact sets in $G$ such that

$$
\lim _{n \rightarrow \infty} \frac{m\left(g F_{n} \triangle F_{n}\right)}{m\left(F_{n}\right)}=0 \quad \text { for all } g \in G .
$$


There are also right Følner sequences which fulfill an analogous condition to (3) where the left Haar measure and the multiplication from the left is replaced by the right Haar measure and multiplication from the right, respectively. From now on, the standard assumption is that we deal with left Haar measures and left $\mathrm{F} \varnothing$ lner sequences if not stated otherwise.

Let $(X, d)$ be a compact metric space. By $\mathcal{C}(X)$ we denote the set of all complex-valued continuous functions on $X$ equipped with the uniform topology which is induced by the sup norm $\|\cdot\|_{\infty}$. Given a Borel probability measure $\mu$ on $X$ and $\varphi \in \mathcal{C}(X)$, we set

$$
\mu(\varphi)=\int \varphi d \mu
$$

The next theorem is well known for $\mathbb{Z}$-actions and can be proven for the group actions considered in this article by adapting the corresponding arguments from [Wal82] and [Fur81]; see also the short discussion regarding Theorem 2.16 in [MR13].

Theorem 2.2: Let $(X, G)$ be a dynamical system. The following statements are equivalent:

(i) $(X, G)$ has a unique $G$-invariant measure $\mu$.

(ii) For each continuous function $\varphi$ on $X$ there is a Følner sequence $\left(F_{n}\right)_{n \in \mathbb{N}}$ with

$$
\lim _{n \rightarrow \infty} \frac{1}{\left|F_{n}\right|} \int_{F_{n}} \varphi(t x) d m(t)=c,
$$

where $c$ is a constant independent of $x \in X$.

Further, if one of the above conditions holds, then the convergence in (ii) is uniform in $x \in X$, independent of the left Følner sequence $\left(F_{n}\right)_{n \in \mathbb{N}}$, and we have

$$
c=\mu(\varphi) .
$$

For the sake of completeness, we provide a proof of the next statement.

Proposition 2.3: Let $(X, G)$ be a dynamical system. Suppose that for each $\varphi \in \mathcal{C}(X)$ there is a right Følner sequence $\left(F_{n}\right)_{n \in \mathbb{N}}$ and a constant $c \in \mathbb{R}$ with

$$
\lim _{n \rightarrow \infty} \frac{1}{\left|F_{n}\right|} \int_{F_{n}} \varphi(t x) d m_{r}(t)=c,
$$

for all $x \in X$. Then $(X, G)$ has a unique $G$-invariant measure $\mu$ and $\mu(\varphi)=c$. 
Proof. As mentioned in the introduction, $(X, G)$ allows for a $G$-invariant measure $\mu$ on $X$. Now, using Fubini and dominated convergence, we have

$$
\begin{aligned}
\int_{X} \varphi d \mu & =\frac{1}{\left|F_{n}\right|} \int_{F_{n}} \int_{X} \varphi(t x) d \mu(x) d m_{r}(t) \\
& =\int_{X} \frac{1}{\left|F_{n}\right|} \int_{F_{n}} \varphi(t x) d m_{r}(t) d \mu(x) \stackrel{n \rightarrow \infty}{\longrightarrow} c .
\end{aligned}
$$

Since finite Borel measures on compact metric spaces are uniquely determined by integrating continuous functions, we obtain that $\mu$ is the only $G$-invariant measure on $X$.

Throughout this work, we will encounter Birkhoff averages of continuous functions, i.e., limits of the above kind, at several places. For that reason, we introduce the following notation: given a left $\mathrm{F} \varnothing$ lner sequence $\mathcal{F}$ and a continuous function $\varphi$ on $X$, we set

$$
A_{n}(\mathcal{F}, \varphi)(x):=\frac{1}{\left|F_{n}\right|} \int_{F_{n}} \varphi(t x) d m(t)
$$

for $x \in X$ and $n \in \mathbb{N}$. Furthermore, we introduce the following functions on $X$

$$
\bar{A}(\mathcal{F}, \varphi): x \mapsto \limsup _{n \rightarrow \infty} A_{n}(\mathcal{F}, \varphi)(x)
$$

and

$$
\underline{A}(\mathcal{F}, \varphi): x \mapsto \liminf _{n \rightarrow \infty} A_{n}(\mathcal{F}, \varphi)(x) .
$$

We simply write $A(\mathcal{F}, \varphi)(x)$ for the above limits, provided they coincide (as in the previous statements). If $\mathcal{F}$ is a right $\mathrm{F} \varnothing$ lner sequence, we refer to the analogous quantities (where the left Haar measure is replaced by the right Haar measure) by the same symbols.

For a dynamical system $(X, G)$ with an ergodic measure $\mu$ and a left $\mathrm{F} \varnothing$ lner sequence $\mathcal{F}$ in $G$, we say a point $x \in X$ is $(\mu$-)generic with respect to $\mathcal{F}$ if for every continuous function $\varphi$ on $X$ the limit $A(\mathcal{F}, \varphi)(x)$ exists and equals $\mu(\varphi)$. It is worth noting and easy to see that every $\mu$-generic point has a dense orbit in the support of $\mu$. For the purpose of being self-contained, we provide a proof of the next well-known statement. Note that a direct consequence of this statement is the well-known singularity of ergodic measures.

Theorem 2.4: Let $(X, G)$ be a topological dynamical system with an ergodic measure $\mu$. Then every left Følner sequence $\mathcal{F}=\left(F_{n}\right)_{n \in \mathbb{N}}$ allows for a subsequence $\mathcal{F}^{\prime}=\left(F_{n}^{\prime}\right)_{n \in \mathbb{N}}$ with respect to which $\mu$-almost every point is generic. 
Proof. Let $\mathcal{F}$ be a left $F \varnothing l n e r$ sequence and $\left(\varphi_{\ell}\right)_{\ell \in \mathbb{N}}$ be a dense sequence in $\mathcal{C}(X)$ (which exists due to Stone-Weierstrass). By the Mean Ergodic Theorem, $A_{n}\left(\mathcal{F}, \varphi_{1}\right)(x) \stackrel{L_{1}}{\longrightarrow} \mu\left(\varphi_{1}\right)$. There is hence a subsequence $\mathcal{F}^{\varphi_{1}}=\left(F_{n}^{\varphi_{1}}\right)_{n \in \mathbb{N}}$ of $\mathcal{F}$ such that

$$
A_{n}\left(\mathcal{F}^{\varphi_{1}}, \varphi_{1}\right)(x) \rightarrow \mu\left(\varphi_{1}\right)
$$

for all $x$ in a full measure set $X_{\varphi_{1}} \subseteq X$. By inductively repeating the above argument, we get that for each $\ell \in \mathbb{N}$ there is a subsequence $\mathcal{F}^{\varphi_{\ell+1}}$ of $\mathcal{F}^{\varphi_{\ell}}$ such that $A_{n}\left(\mathcal{F}^{\varphi_{\ell+1}}, \varphi_{\ell+1}\right)(x) \rightarrow \mu\left(\varphi_{\ell+1}\right)$ for all $x$ in a full measure set $X_{\varphi_{\ell+1}} \subseteq X$. Set

$$
X_{\mathcal{C}(X)}=\bigcap_{\ell \in \mathbb{N}} X_{\varphi_{\ell}} \quad \text { and } \quad \mathcal{F}^{\prime}=\left(F_{n}^{\varphi_{n}}\right)_{n \in \mathbb{N}} .
$$

Clearly, we have

$$
\mu\left(X_{\mathcal{C}(X)}\right)=1
$$

Moreover,

$$
\bar{A}\left(\mathcal{F}^{\prime}, \varphi_{\ell}\right)(x)=\underline{A}\left(\mathcal{F}^{\prime}, \varphi_{\ell}\right)(x)=\mu\left(\varphi_{\ell}\right)
$$

for all $\ell \in \mathbb{N}$ and $x \in X_{\mathcal{C}(X)}$. Note that for every fixed $x \in X$ we have that $\bar{A}\left(\mathcal{F}^{\prime}, \varphi\right)(x), \underline{A}\left(\mathcal{F}^{\prime}, \varphi\right)(x)$, and $\mu(\varphi)$ depend continuously on $\varphi \in \mathcal{C}(X)$. Altogether, we thus have for every $\varphi \in \mathcal{C}(X)$ and every $x \in X_{\mathcal{C}(X)}$ that

$$
\begin{aligned}
\bar{A}\left(\mathcal{F}^{\prime}, \varphi\right)(x) & =\lim _{j \rightarrow \infty} \bar{A}\left(\mathcal{F}^{\prime}, \varphi_{\ell_{j}}\right)(x)=\lim _{j \rightarrow \infty} \underline{A}\left(\mathcal{F}^{\prime}, \varphi_{\ell_{j}}\right)(x) \\
& =\underline{A}\left(\mathcal{F}^{\prime}, \varphi\right)(x) \\
& =\lim _{j \rightarrow \infty} \mu\left(\varphi_{\ell_{j}}\right)=\mu(\varphi),
\end{aligned}
$$

where $\left(\varphi_{\ell_{j}}\right)_{j \in \mathbb{N}}$ is a subsequence of $\left(\varphi_{\ell}\right)_{\ell \in \mathbb{N}}$ with $\varphi_{\ell_{j}} \rightarrow \varphi$.

We will need the following auxiliary statement which is immediately linked to the ergodic representation of invariant measures; see, for instance, [Far62] for more information.

Lemma 2.5 ([Far62, Lemma 6]): Let $\mu$ be a $G$-invariant measure. If $\mu(A)>0$ for some Borel measurable set $A \subseteq X$, then there is an ergodic $G$-invariant measure $\nu$ with $\nu(A)>0$. 


\section{Topo-isomorphic extensions}

In the following we establish the equivalence of (Weyl-) mean equicontinuity and topo-isomorphy and thus prove our main structural result (Theorem 1.1). To that end, we first gather some basics on topo-isomorphic extensions in Subsection 3.1. Then Theorem 3.7 (in Subsection 3.2) yields one direction of the main theorem. Theorem 3.15 (in Subsection 3.3) yields the other direction.

Theorem 1.1 naturally suggests to also look at the relation between mean equicontinuous systems and their topo-isomorphic extensions. We show that the preservation of the maximal equicontinuous factor is a characteristic of such extensions (see Subsection 3.4).

3.1. BASICS ON TOPO-ISOMORPHIC EXTEnsions. In this section we explain the structure of topo-isomorphic extensions over equicontinuous systems. Roughly speaking, such systems are partitioned into uniquely ergodic components and this will be relevant in our considerations hereafter.

Proposition 3.1: Suppose $(X, G)$ is a topo-isomorphic extension of $(Y, G)$ via the factor map $h: X \rightarrow Y$. If $\mu_{1}$ and $\mu_{2}$ are two distinct ergodic $G$-invariant measures on $X$, then the image measures $h\left(\mu_{1}\right)$ and $h\left(\mu_{2}\right)$ differ as well.

Proof. Assume for a contradiction that there exist distinct ergodic $G$-invariant measures $\mu_{1}$ and $\mu_{2}$ such that $h\left(\mu_{1}\right)=h\left(\mu_{2}\right)$. Since $h$ is a topo-isomorphy, $h\left(\mu_{1}\right)$ is ergodic. Now, consider $\mu=1 / 2 \cdot\left(\mu_{1}+\mu_{2}\right)$. Clearly, $\mu$ is not ergodic, since it is a convex combination of two distinct ergodic measures. Since $h$ is a topo-isomorphy, $h(\mu)$ is not ergodic, too. This contradicts

$$
h(\mu)=1 / 2 \cdot\left(h\left(\mu_{1}\right)+h\left(\mu_{2}\right)\right)=h\left(\mu_{1}\right) .
$$

We will make use of the following classical lemma (see, for example, [Aus88]) which gives that the notions of transitivity and minimality coincide for equicontinuous systems.

Lemma 3.2: If $(X, G)$ is equicontinuous, then for each $x \in X$ we have that $\overline{G x}$ is minimal.

Regarding the next statements, see also Theorem 14 (Decomposition Theorem) in [Aus59] for the case of $\mathbb{Z}$-actions. 
Proposition 3.3: Assume that $(X, G)$ is a topo-isomorphic extension of an equicontinuous system $(Y, G)$ with factor map $h$.

(a) If $A \subseteq X$ is transitive, then $h(A)$ is minimal. In particular, if $B$ is another transitive subset of $X$, then either $h(A)=h(B)$ or $h(A) \cap h(B)=\emptyset$.

(b) Let $A$ be a closed $G$-invariant subset of $X$. The set $h(A)$ is minimal if and only if $A$ is uniquely ergodic.

Proof. (a) Since factor maps preserve transitivity, this follows from Lemma 3.2.

(b) Suppose $h(A)$ is minimal. Since $(Y, G)$ is equicontinuous, minimal subsets are uniquely ergodic (this classical fact also follows from Theorem 5.6 below). Hence, $h$ maps every invariant measure on $A$ to the same invariant measure on $h(A)$. By Proposition 3.1, $A$ is uniquely ergodic.

Conversely, suppose $A$ is uniquely ergodic. As any orbit closure carries an invariant measure, $\overline{G x}$ and $\overline{G y}$ have a non-empty intersection for $x, y \in A$. Now, (a) yields

$$
h(\overline{G x})=h(\overline{G y}) \quad(x, y \in A) .
$$

As $x$ and $y$ are arbitrary elements of $A$, this shows that $h(A)$ coincides with the image of the transitive set $\overline{G x}$ under $h$. Due to (a), $h(A)$ is hence minimal.

Theorem 3.4: Assume that $(X, G)$ is a topo-isomorphic extension of an equicontinuous system $(Y, G)$ with factor map $h: X \rightarrow Y$. Then the following statements are true.

(a) $(X, G)$ is pointwise uniquely ergodic.

(b) If $\mu$ and $\nu$ are distinct ergodic measures on $X$ supported on transitive sets $A_{\mu}$ and $A_{\nu}$, respectively, then

$$
h\left(A_{\mu}\right) \cap h\left(A_{\nu}\right)=\emptyset .
$$

Proof. (a) Clearly $\overline{G x}$ is a transitive subset of $X$ for any $x \in X$. The statement follows from (a) and (b) of the previous proposition.

(b) By (a) of the previous proposition, we either have $h\left(A_{\mu}\right)=h\left(A_{\nu}\right)$ or $h\left(A_{\mu}\right) \cap h\left(A_{\nu}\right)=\emptyset$. So, it remains to show that $h\left(A_{\mu}\right)=h\left(A_{\nu}\right)$ is not possible. To that end, assume the contrary. Then,

$$
C:=h^{-1}\left(h\left(A_{\mu}\right)\right)=h^{-1}\left(h\left(A_{\nu}\right)\right)
$$

is a closed invariant subset of $X$ that contains $A_{\mu}$ and $A_{\nu}$. Hence, it is not uniquely ergodic. This contradicts the previous proposition. 
As a consequence of the preceding theorem we can decompose topo-isomorphic extensions of equicontinuous systems into uniquely ergodic components. Let us introduce the following notation: whenever $(X, G)$ is a dynamical system and $\mu$ an ergodic measure, $X_{\mu}$ denotes the set of all $x \in X$ whose orbit closure $\overline{G x}$ supports $\mu$ and no other invariant measure. In other words, $X_{\mu}$ comprises the set of all points which are $\mu$-generic with respect to each Følner sequence.

Corollary 3.5: Let $(X, G)$ be a topo-isomorphic extension of an equicontinuous system $(Y, G)$. Then the sets $X_{\mu}$ partition $X$, that is $X=\bigsqcup_{\mu} X_{\mu}$, where $\mu$ runs over all ergodic measures on $X$. Further, each $X_{\mu}$ is the preimage of a minimal subset of $Y$ and any such preimage coincides with an $X_{\mu}$.

Proof. According to the previous theorem, $(X, G)$ is pointwise uniquely ergodic which immediately gives that the sets $X_{\mu}$ partition $X$.

For the second part, Lemma 3.2 yields that it suffices to show that to each minimal set $M \subseteq Y$ there is a unique ergodic measure $\mu$ on $X$ such that $h^{-1}(M)=X_{\mu}$, with $h$ the factor map from $X$ to $Y$. Proposition 3.3 (b) yields that there is a unique ergodic measure $\mu$ on $X$ with $h^{-1}(M) \subseteq X_{\mu}$. Clearly, for any $x \in X$ whose orbit closure supports $\mu$, we must have $\overline{G x} \cap h^{-1}(M) \neq \emptyset$. Now, due to Proposition 3.3 (a), $h(\overline{G x})$ is minimal which necessarily yields $x \in h^{-1}(M)$.

Corollary 3.6: Suppose $(X, d)$ is a compact, connected metric space and $(X, G)$ is mean equicontinuous. Then $(X, G)$ has either a unique ergodic measure (minimal set) or uncountably many ergodic measures (minimal sets).

Proof. Recall that the support of an ergodic measure is always transitive (due to the generic points) and that every minimal set supports an ergodic measure. Due to the pointwise unique ergodicity of mean equicontinuous systems (see Theorem 3.15 and Theorem 3.4 (a)), this implies that there is a one-to-one correspondence between minimal sets and ergodic measures. Hence, it suffices to show the statement for ergodic measures.

Due to Corollary 3.5, we have a bijection between the ergodic measures of $(X, G)$ and the minimal sets of its maximal equicontinuous factor $(\mathbb{T}, G)$. By Lemma 3.2, $\mathbb{T}$ allows for a partition by minimal sets which are clearly compact and pairwise disjoint. Since $\mathbb{T}$ is connected (as the continuous image of $X$ ), a classical result by Sierpinski [Sie18] yields that such a partition consists of either one or uncountably many partition elements. 
3.2. Topo-ISOMORPhy implies MeAn Equicontinuity. Now, we show one direction of our main structural result. To that end, we make use of Proposition 2.1 and rephrase the assertion that topo-isomorphy implies mean equicontinuity as follows.

Theorem 3.7: Let $(X, G)$ be a dynamical system and $\left(\mathbb{T}^{\prime}, G\right)$ an equicontinuous factor with factor map $\pi^{\prime}$ such that for every $G$-invariant measure $\mu$ the operator

$$
U_{\mu}: L_{2}\left(\mathbb{T}^{\prime}, \pi(\mu)\right) \rightarrow L_{2}(X, \mu): f \mapsto f \circ \pi^{\prime}
$$

is unitary. Then $(X, G)$ is mean equicontinuous and $\left(\mathbb{T}^{\prime}, G\right)$ is the associated MEF.

Before we can turn to the proof of Theorem 3.7, we need two further ingredients. The first ingredient is another characterization of mean equicontinuity which makes use of the continuous functions on $X$. For that purpose we define the pseudometric $D_{f}$ associated to a function $f \in \mathcal{C}(X)$ by

$D_{f}(x, y):=\sup \left\{\varlimsup_{n \rightarrow \infty} \frac{1}{\left|F_{n}\right|} \int_{F_{n}}|f(t x)-f(t y)| d m(t) \mid\left(F_{n}\right)_{n \in \mathbb{N}}\right.$ a Følner sequence $\}$.

The following statement is well-known; see [DI88, Proposition 1] and [GRM19, Theorem 2.14]. We include a proof for the convenience of the reader.

Proposition 3.8: The following assertions are equivalent:

(i) $(X, G)$ is mean equicontinuous.

(ii) For every $f \in \mathcal{C}(X)$ the pseudometric $D_{f}$ is continuous.

Moreover, if one of the equivalent assertions (i) and (ii) holds, then $D(x, y)=0$ if and only if $D_{f}(x, y)=0$ for all continuous $f$.

Proof. It is not hard to see that the topology generated on $X$ by $D$ as well as the mean equicontinuity of $(X, G)$ is independent of the particular choice of the metric $d$ (provided $d$ generates the original topology on $X$ ). This will be used throughout the proof.

(i) $\Rightarrow$ (ii): Observe that

$$
d^{\prime}(x, y):=d(x, y)+|f(x)-f(y)|
$$

is a metric equivalent to $d$. We can hence assume w.l.o.g. that

$$
|f(x)-f(y)| \leq d(x, y) .
$$

This implies $D_{f} \leq D$. As $D$ is continuous, this implies (ii). 
(ii) $\Rightarrow$ (i): Choose a sequence $\left(f_{n}\right)_{n \in \mathbb{N}}$ of continuous functions on $X$ which separate points and satisfy $\left\|f_{n}\right\|_{\infty} \leq 1$ for all $n \in \mathbb{N}$. Then for any $c_{n}>0$ with $\sum_{n} c_{n}<\infty$ we have that

$$
\sum_{n} c_{n}\left|f_{n}(x)-f_{n}(y)\right|
$$

defines a metric equivalent to $d$. We can hence assume w.l.o.g. that $d$ is given by (4). Now, clearly

$$
D \leq \sum c_{n} D_{f_{n}}=: \widetilde{D},
$$

where $\widetilde{D}$ is continuous by (ii) and the summability of $\left(c_{n}\right)_{n \in \mathbb{N}}$.

The last statement has been shown along the proof.

Remark 3.9: The above shows that the topology on $X$ generated by $D$ agrees with the topology generated by the collection of $D_{f}$ 's with $f \in \mathcal{C}(X)$.

The other ingredient needed for the proof of Theorem 3.7 -and in some sense the main insight of the present section- is the following lemma.

Lemma 3.10: Let $(X, G)$ be a dynamical system and $\left(\mathbb{T}^{\prime}, G\right)$ an equicontinuous factor with factor map $\pi^{\prime}$. Suppose that for every $G$-invariant measure $\mu$ the operator

$$
U_{\mu}: L_{2}\left(\mathbb{T}^{\prime}, \pi^{\prime}(\mu)\right) \rightarrow L_{2}(X, \mu): f \mapsto f \circ \pi^{\prime}
$$

is unitary. Then, for any $f \in \mathcal{C}(X)$ and any $\varepsilon>0$ we have $D_{f}\left(x_{1}, x_{2}\right)<\varepsilon$ provided $\pi^{\prime}\left(x_{1}\right)$ and $\pi^{\prime}\left(x_{2}\right)$ are sufficiently close.

Proof. By (a) of Theorem 3.4 the orbit closure of $x_{i}(i=1,2)$ supports a unique ergodic measure $\mu_{i}$. W.l.o.g. we may assume that $\mu_{1} \neq \mu_{2}$ (if $\mu_{1}=\mu_{2}$, the following argument works in an analogous and slightly simplified way). By unitarity of the $U_{\mu_{i}}$ 's and denseness of continuous functions in $L_{2}\left(\mathbb{T}^{\prime}, \pi^{\prime}\left(\mu_{i}\right)\right)$, we can find $g_{i} \in \mathcal{C}\left(\mathbb{T}^{\prime}\right)$ with

$$
\left\|f-g_{i} \circ \pi^{\prime}\right\|_{L_{2}\left(X, \mu_{i}\right)}=\left\|f-U_{\mu_{i}} g_{i}\right\|_{L_{2}\left(X, \mu_{i}\right)} \leq \varepsilon / 3 .
$$

By Cauchy-Schwarz, we then obtain

$$
\left\|f-g_{i} \circ \pi^{\prime}\right\|_{L_{1}\left(X, \mu_{i}\right)} \leq\left\|f-g_{i} \circ \pi^{\prime}\right\|_{L_{2}\left(X, \mu_{i}\right)} \leq \varepsilon / 3 .
$$

Set $M_{i}=\pi^{\prime}\left(\overline{G x_{i}}\right)$. Then $M_{1}$ and $M_{2}$ are disjoint by Theorem 3.4 (b) since $\mu_{1} \neq \mu_{2}$. Let $S_{i}(i=1,2)$ be continuous functions on $\mathbb{T}^{\prime}$ with $\left.S_{i}\right|_{M_{i}}=1$ and $\left.S_{1}\right|_{M_{2}}=\left.S_{2}\right|_{M_{1}}=0$. Set $g=S_{1} g_{1}+S_{2} g_{2}$. 
Now, for any $t \in G$ we have

$$
\begin{aligned}
\left|f\left(t x_{1}\right)-f\left(t x_{2}\right)\right| & \leq\left|f\left(t x_{1}\right)-g_{1} \circ \pi^{\prime}\left(t x_{1}\right)\right|+\left|g \circ \pi^{\prime}\left(t x_{1}\right)-g \circ \pi^{\prime}\left(t x_{2}\right)\right| \\
& +\left|g_{2} \circ \pi^{\prime}\left(t x_{2}\right)-f\left(t x_{2}\right)\right| .
\end{aligned}
$$

Consequently, we obtain

$$
D_{f}\left(x_{1}, x_{2}\right) \leq T_{1}\left(x_{1}\right)+D_{g \circ \pi^{\prime}}\left(x_{1}, x_{2}\right)+T_{2}\left(x_{2}\right),
$$

where

$$
\begin{aligned}
T_{i}\left(x_{i}\right):=\sup \left\{\varlimsup_{n \rightarrow \infty} \frac{1}{\left|F_{n}\right|} \int_{F_{n}}\left|f\left(t x_{i}\right)-g_{i} \circ \pi^{\prime}\left(t x_{i}\right)\right| d m(t)\right. \\
\left.\qquad \mid\left(F_{n}\right)_{n \in \mathbb{N}} \text { a Følner sequence }\right\} .
\end{aligned}
$$

We show that all three terms become small for $x_{1}$ sufficiently close to $x_{2}$. By unique ergodicity on orbit closures and Theorem 2.2 , we obtain

$$
T_{i}\left(x_{i}\right)=\left\|f-g_{i} \circ \pi^{\prime}\right\|_{L_{1}\left(X, \mu_{i}\right)} \leq \varepsilon / 3 .
$$

The term $D_{g \circ \pi^{\prime}}$ can be treated as follows. If $\pi^{\prime}\left(x_{1}\right)$ is close to $\pi^{\prime}\left(x_{2}\right)$, we obtain that $t \pi^{\prime}\left(x_{1}\right)$ is close to $t \pi^{\prime}\left(x_{2}\right)$ for all $t \in G$ (by equicontinuity). As $g$ is continuous (and hence uniformly continuous) on $\mathbb{T}^{\prime}$, this implies that $g \circ \pi^{\prime}\left(t x_{1}\right)=g\left(t \pi^{\prime}\left(x_{1}\right)\right)$ is close to $g \circ \pi^{\prime}\left(t x_{2}\right)=g\left(t \pi^{\prime}\left(x_{2}\right)\right)$ for all $t \in G$ and we are done.

Proof of Theorem 3.7. We first show that $(X, G)$ is mean equicontinuous. By Proposition 3.8, it suffices to show that $D_{f}$ is continuous for any $f \in \mathcal{C}(X)$. Let such an $f$ be given and consider an arbitrary $\varepsilon>0$. We have to show that if $x_{1}, x_{2} \in X$ are close, then $D_{f}\left(x_{1}, x_{2}\right)<\varepsilon$. This, however, is clear from Lemma 3.10 as for $x_{1}$ close to $x_{2}$ we clearly have $\pi^{\prime}\left(x_{1}\right)$ close to $\pi^{\prime}\left(x_{2}\right)$ due to the continuity of $\pi^{\prime}$.

It remains to show that $\left(\mathbb{T}^{\prime}, G\right)$ is the MEF. As discussed in Subsection 1.1, it suffices to show that $\inf _{t \in G} d(t x, t y)=0$ whenever $\pi^{\prime}(x)=\pi^{\prime}(y)$. Now, $\pi^{\prime}(x)=\pi^{\prime}(y)$ implies $D_{f}(x, y)=0$ for all continuous $f$ on $X$ (by Lemma 3.10) and hence $D(x, y)=0$ due to Proposition 3.8. From this and the definition of $D$ we easily find

$$
\inf _{t \in G} d(t x, t y)=0
$$




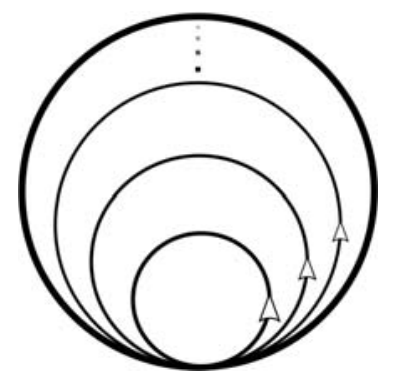

Figure 1. A sketch of a $\mathbb{Z}$-action which is not mean equicontinuous but at the same time a pointwise uniquely ergodic topo-isomorphic extension of its trivial MEF with respect to all ergodic (delta) measures. The continuous dynamics are as follows: points on the bold outer circle are fixed. Further, there are infinitely many inner circles attached to the south pole which accumulate at the outer circle and all points on the inner circles get attracted by the south pole.

Remark 3.11: We defined a topo-isomorphy $h$ to be a topological factor map which is an isomorphism $(\bmod 0)$ with respect to $\mu$ and $h(\mu)$ for every invariant measure $\mu$. It is natural to ask whether Theorem 3.7 still remains true if we relax the assumptions on $h$ by considering $h$ to be a factor map which is only an isomorphism $(\bmod 0)$ with respect to $\mu$ and $h(\mu)$ for every ergodic measure $\mu$. In the proof of Lemma 3.10, the topo-isomorphy with respect to every invariant measure was (implicitly) used twice: once, to ensure pointwise unique ergodicity and once, to ensure that the supports of two distinct ergodic measures have disjoint images under $h$ (see also Theorem 3.4). In fact, the latter implies the former and hence implies mean equicontinuity if we additionally assume $h$ to be a topo-isomorphy onto an equicontinuous factor with respect to every ergodic measure. However, it is not true that pointwise unique ergodicity and topoisomorphy to an equicontinuous factor with respect to ergodic measures only yields that the supports of distinct ergodic measures have distinct images, as can be seen in Figure 1. 
3.3. MEAN EQUiCONTINUITY IMPLIES TOPO-ISOMORPHY. In this section we establish that mean equicontinuity implies topo-isomorphy of the dynamical system to its MEF. Together with Theorem 3.7 from the previous subsection, this proves our main structural result Theorem 1.1. We first note that $D$ is actually $G$-invariant.

Proposition 3.12 (Invariance of $D$ ): Let $(X, G)$ be a dynamical system. Then $D$ satisfies

$$
D(t x, t y)=D(x, y)
$$

for all $x, y \in X$ and $t \in G$.

Proof. Recall that there exists a unique $\Delta: G \rightarrow(0, \infty)$ (called modular function) whose defining property is that

$$
\int h(t s) d m(t)=\Delta(s) \int h(t) d m(t),
$$

for all Haar measurable $h: G \rightarrow[0, \infty)$. A short computation and canceling of modular functions then gives that

$$
\frac{1}{|F s|} \int_{F s} g(t) d m(t)=\frac{1}{|F|} \int_{F} g(t s) d m(t),
$$

for all $s \in G$ and all Haar measurable bounded $g: G \rightarrow[0, \infty)$ whenever $F$ is a compact subset of $G$ with positive Haar measure. This shows that

$$
D_{\mathcal{F}}(s x, s y)=D_{\mathcal{F} s}(x, y),
$$

where $\mathcal{F} s$ denotes the sequence $\left(F_{n} s\right)_{n \in \mathbb{N}}$. Now, $\left(F_{n} s\right)_{n \in \mathbb{N}}$ is clearly a Følner sequence as well. Hence, the desired statement follows as the definition of $D$ involves all Følner sequences.

Remark 3.13: For later reference, recall that the group $G$ is referred to as unimodular if the modular function $\Delta$ is identically 1 , that is, $G$ is unimodular if and only if left Haar measures are also right Haar measures (and vice versa).

Let a dynamical system $(X, G)$ be given. For $x, y \in X$ write $x \sim y$ if $D(x, y)=0$. If $(X, G)$ is mean equicontinuous, then clearly the quotient map $\beta: X \rightarrow X / \sim$ is continuous. By the invariance of $D$ due to Proposition 3.12, the action of $G$ on $X / \sim$ given by $g \beta(x):=\beta(g x)$ is well defined and isometric. Hence, $(X / \sim, G)$ is an equicontinuous factor of $(X, G)$. 
Proposition 3.14: If $(X, G)$ is mean equicontinuous, then $(X / \sim, G)$ is its MEF.

Proof. As discussed in Section 1.1 it suffices to show that $\inf _{t \in G} d(t x, t y)=0$ whenever $\beta(x)=\beta(y)$. This, however, is clear.

As pointed out in the introduction, the proof of the next statement is inspired - and to a large extent similar - to the proof of [LTY15, Theorem 3.8].

Theorem 3.15: Suppose $(X, G)$ is mean equicontinuous. Then $(X, G)$ is topoisomorphic to its maximal equicontinuous factor $(\mathbb{T}, G)$.

Proof. Fix a $G$-invariant measure $\mu$ and let $\mu=\int \mu_{z} d \nu(z)$ be the disintegration of $\mu$ over its image measure $\nu:=\pi(\mu)$ (see, e.g., [Fur81]). We consider the relative product measure $\mu \times_{\nu} \mu$ supported in the relative product of $X$ over $\mathbb{T}$

$$
X \times_{\mathbb{T}} X:=\{(x, y) \in X \times X \mid \pi(x)=\pi(y)\}
$$

which is defined by

$$
\mu \times{ }_{\nu} \mu:=\int \mu_{z} \times \mu_{z} d \nu(z) .
$$

Recall that $\mu \times_{\nu} \mu$ is invariant under the action of $G$ on $X \times_{\mathbb{T}} X$ given by

$$
g(x, y):=(g x, g y)
$$

for each $(x, y) \in X \times_{\mathbb{T}} X$ and $g \in G$; see Proposition 5.14 in [Fur81].

We claim that $\mu \times_{\nu} \mu$ is only supported on the diagonal

$$
\{(x, x) \in X \times X \mid x \in X\} \subseteq X \times_{\mathbb{T}} X .
$$

For a contradiction assume this is not the case. Then there exists an open set $A$ in $X \times_{\mathbb{T}} X$ which has a positive distance to the diagonal and fulfills

$$
\left(\mu \times{ }_{\nu} \mu\right)(A)>0 .
$$

Using Lemma 2.5, this yields that there is an ergodic measure $\tilde{\mu}$ on $X \times_{\mathbb{T}} X$ with $\tilde{\mu}(A)>0$. According to Theorem 2.4, $\tilde{\mu}$-almost every point is $\tilde{\mu}$-generic with respect to some $\mathrm{F} \varnothing$ lner sequence $\mathcal{F}$. Now, for every such $(x, y) \in X \times_{\mathbb{T}} X$ we have

$$
D(x, y) \geq D_{\mathcal{F}}(x, y)=\int d(z, w) d \tilde{\mu}(z, w)>0 .
$$

This is in contradiction to the previous proposition because

$$
\pi(x)=\pi(y) \Leftrightarrow D(x, y)=0
$$

in case that $(X, G)$ is mean equicontinuous. 
Now, observe that the only measures supported in $\pi^{-1}(z)$ whose Cartesian squares are supported in the diagonal of $X \times_{\mathbb{T}} X$ are delta measures. Thus, $\mu_{z}$ is a delta measure for $\nu$-almost every $z \in \mathbb{T}$. Finally, the map which assigns to each $z$ the support of $\mu_{z}$ is an isomorphism with respect to $\nu$ and $\mu$ whose inverse coincides with $\pi$ for $\mu$-a.e. point.

3.4. Further properties and first non-minimal examples. Here, we discuss first consequences of the results of the previous subsections. In particular, we show that the preservation of the maximal equicontinuous factor is a characteristic feature of topo-isomorphic extensions of mean equicontinuous systems. Furthermore, we discuss some examples of non-minimal mean equicontinuous systems for $G=\mathbb{Z}$.

ThEOREM 3.16 (Characterization of mean equicontinuous extensions): Let $(X, G)$ be an extension of a mean equicontinuous system $(Y, G)$. Then, $(X, G)$ is topo-isomorphic to $(Y, G)$ if and only if it is mean equicontinuous and its $M E F$ agrees with that of $(Y, G)$.

Proof. Assume first that $(X, G)$ is a topo-isomorphic extension of $(Y, G)$. By Theorem 3.15, $(Y, G)$ is a topo-isomorphic extension of its MEF ( $\mathbb{T}, G)$. Clearly, $(X, G)$ is also a topo-isomorphic extension of $(\mathbb{T}, G)$. The statement now follows from Theorem 3.7 .

Consider now the situation that $(X, G)$ is mean equicontinuous and the MEFs of $(Y, G)$ and $(X, G)$ agree. Let $h$ be the factor map from $X$ to $Y$ and $\pi$ a factor map from $Y$ to $\mathbb{T}$ (the MEF of both systems). Note that both $\pi$ and $\pi \circ h: X \rightarrow \mathbb{T}$ are topo-isomorphies, according to Theorem 3.15. This implies that $h$ is a topo-isomorphy, too.

Corollary 3.17: If two equicontinuous dynamical systems $(X, G)$ and $(Y, G)$ are topo-isomorphic, then they are in fact topological conjugate.

Proof. By the previous theorem such systems share the same MEF. By equicontinuity, however, they agree with their MEF.

Remark 3.18: The corollary is reminiscent of the rigidity phenomenon which is well known for ergodic abelian equicontinuous group actions, see for instance [FK02].

In order to state another consequence of Theorem 3.16 we need the following observation. 
Proposition 3.19: Any topological factor of a mean equicontinuous system is mean equicontinuous as well.

Proof. Let $(Y, G)$ be a factor of a mean equicontinuous system $(X, G)$ with factor map $h$. By Proposition 3.8, it suffices to show that $D_{f}$ is continuous for any continuous $f$ on $Y$.

First, observe that Proposition 3.8 ensures continuity of $D_{f \circ h}$. Now, continuity of $D_{f}$ follows from continuity of $D_{f \circ h}$. For a contradiction, suppose we are given some $\varepsilon>0$ and a sequence $\left(y_{n}\right)_{n \in \mathbb{N}}$ in $Y$ which converges to some $y \in Y$ while $D_{f}\left(y_{n}, y\right)>\varepsilon$. Since $h$ is surjective (by the definition of factor maps), we find a sequence $\left(x_{n}\right)_{n \in \mathbb{N}}$ in $X$ with $h\left(x_{n}\right)=y_{n}$ for each $n \in \mathbb{N}$. As $X$ is compact, we may assume without loss of generality that $\left(x_{n}\right)_{n \in \mathbb{N}}$ converges to some $x \in X$. By continuity of $h, h(x)=y$. The continuity of $D_{f \circ h}$ hence gives

$$
D_{f}\left(y_{n}, y\right)=D_{f}\left(h\left(x_{n}\right), h(x)\right)=D_{f \circ h}\left(x_{n}, x\right) \rightarrow 0
$$

which contradicts $D_{f}\left(y_{n}, y\right)>\varepsilon$. The statement follows.

Given this proposition, Theorem 3.16 has the following immediate consequence (systems fitting into the setting of the following statement can be found in [DD02, Section 5]).

Corollary 3.20: If $(X, G)$ is mean equicontinuous and an extension of $(Y, G)$ with the same $M E F$, then $(X, G)$ is a topo-isomorphic extension of $(Y, G)$.

Much of the previous work on mean equicontinuity is concerned with minimal $\mathbb{Z}$-actions. Therefore, we would like to close this section with a discussion of two simple kinds of examples of well-known systems which are mean equicontinuous but not minimal. The first example will be still transitive (in fact, as we will see, all but one point have a dense orbit) and the second kind of examples will have no dense orbits but will still be uniquely ergodic. For a non-uniquely ergodic system, see Example 5.11.

Example 3.21: Consider the Cantor substitution

$$
0 \mapsto 010 \text { and } 1 \mapsto 111
$$

For a general introduction to substitution systems, see for example [Ků03]. There are two infinite sequences in $\{0,1\}^{\mathbb{N}}$ which are invariant with respect to the Cantor substitution: the constant sequence $(111 \ldots)$ and the sequence $\omega$ 
obtained by applying the substitution successively to the letter 0 and its images, i.e.,

$$
0 \mapsto 010 \mapsto 010111010 \mapsto 010111010111111111010111010 \mapsto \cdots .
$$

Now, there is a standard method to obtain a two-sided subshift $\left(\Sigma_{\omega}, \sigma\right)$ from $\omega$; see, for instance, [Ku03, Proposition 3.71]. That is, $\Sigma_{\omega}$ is a closed subset of $\{0,1\}^{\mathbb{Z}}$ (equipped with the product topology) which is invariant under the action of the left shift $\sigma:\{0,1\}^{\mathbb{Z}} \rightarrow\{0,1\}^{\mathbb{Z}}$. By making use of the concrete structure of $\omega$, it is not difficult to see that all points in $\Sigma_{\omega}$, except the constant sequence $(\ldots 111 \ldots)$, have a dense orbit and that the letter 0 occurs with zero density in each sequence of $\Sigma_{\omega}$. The former implies that $\Sigma_{\omega}$ is uncountable and the latter that $\left(\Sigma_{\omega}, \sigma\right)$ is uniquely ergodic, with the unique invariant measure the delta measure supported on $(\ldots 111 \ldots)$. Thus $\left(\Sigma_{\omega}, \sigma\right)$ is a non-trivial topo-isomorphic extension of its trivial MEF and hence, mean equicontinuous.

Example 3.22: By a classical result of Denjoy [Den32], there exist examples of $C^{1}$ circle diffeomorphisms which have a rigid rotation $\left(\mathbb{S}^{1}, R_{\alpha}\right)$, with $\alpha \in \mathbb{R}$ irrational, as a factor but are not conjugate to it. Herman [Her79] showed later that these examples can even be made $C^{1+\varepsilon}$ for any $\varepsilon<1$. We will refer to these kind of systems as Denjoy examples.

All Denjoy examples have a unique minimal set $C \subset \mathbb{S}^{1}$ and a unique invariant measure $\mu$ supported on $C$. We claim that any Denjoy system $\left(\mathbb{S}^{1}, f\right)$ is mean equicontinuous because of the following reason. Since the factor map $\pi: \mathbb{S}^{1} \rightarrow \mathbb{S}^{1}$, extending $\left(\mathbb{S}^{1}, R_{\alpha}\right)$ to $\left(\mathbb{S}^{1}, f\right)$, is monotone, we have that $\pi^{-1}(\theta)$ for $\theta \in \mathbb{S}^{1}$ is either a singleton or an interval. This immediately implies that the set of non-invertible points

$$
\left\{\theta \in \mathbb{S}^{1}: \# \pi^{-1}(\theta)>1\right\}
$$

is countable. Accordingly, we get that $\pi$ is invertible on a full measure set with respect to $\mu$ (since $\pi(\mu)$ is the Lebesgue measure on $\mathbb{S}^{1}$, the unique invariant measure of $R_{\alpha}$ ).

McSwiggen has shown that there are Denjoy homeomorphisms on higherdimensional tori that share the same properties just mentioned, in particular, that the set of non-invertible points is countable. This means these systems are mean equicontinuous, too. For examples on the two-torus, see [McS93] as well as [NV94, NS96] for more information concerning these systems. For examples defined on general $k$-tori, $k \geq 2$, see [McS95]. 


\section{Mean equicontinuity via product systems}

In Theorem 3.4 we have seen that any mean equicontinuous system is pointwise uniquely ergodic. Here, we show that pointwise unique ergodicity of the product system together with a continuity property is an equivalent characterization of mean equicontinuity.

Proposition 4.1: If there is a left (or right) Følner sequence $\mathcal{F}=\left(F_{n}\right)_{n \in \mathbb{N}}$ so that $(X, G)$ is $\mathcal{F}$-mean equicontinuous, then for each $\varphi \in \mathcal{C}(X), \bar{A}(\mathcal{F}, \varphi)(\cdot)$ and $\underline{A}(\mathcal{F}, \varphi)(\cdot)$ are continuous.

Proof. Similarly as in the proof of Proposition 3.8 (i) $\Rightarrow$ (ii), we see that for each $\varepsilon>0$ there is $\delta>0$ such that

$$
\varlimsup_{n \rightarrow \infty} \frac{1}{\left|F_{n}\right|} \int_{F_{n}}|\varphi(t x)-\varphi(t y)| d m(t)<\varepsilon,
$$

whenever $d(x, y)<\delta$. This immediately gives the continuity of $\bar{A}(\mathcal{F}, \varphi)$ and $\underline{A}(\mathcal{F}, \varphi)$.

In the following, if $(X, G)$ is pointwise uniquely ergodic, then the map $x \mapsto \mu_{x}$ from $X$ into the space of all Borel probability measures on $X$ (equipped with the weak-*topology) is defined to send each $x \in X$ to the unique $G$-invariant measure $\mu_{x}$ supported on $\overline{G x}$. The next statement provides an extension of [LTY15, Theorem 3.3].

THEOREM 4.2: For a system $(X, G)$ the following conditions are equivalent:

(i) $(X, G)$ is mean equicontinuous.

(i) $(X \times X, G)$ is pointwise uniquely ergodic and the map $(x, y) \mapsto \mu_{(x, y)}$ is continuous.

Proof. First, assume that $(X, G)$ is mean equicontinuous. This easily implies that the product system $(X \times X, G)$ is also mean equicontinuous. According to Theorem 3.4 (a), this in turn yields that $(X \times X, G)$ is pointwise uniquely ergodic. Now, consider some left Følner sequence $\mathcal{F}$. From the previous proposition we have that for every $\varphi \in \mathcal{C}(X \times X)$ the functions $\underline{A}(\mathcal{F}, \varphi)(\cdot)$ and $\bar{A}(\mathcal{F}, \varphi)(\cdot)$ are continuous on $X \times X$. Hence, using Theorem 2.2, for a sequence of points $\left(x_{n}, y_{n}\right) \in X \times X$ converging to $(x, y)$ as $n \rightarrow \infty$ we have

$$
\lim _{n \rightarrow \infty} \int \varphi d \mu_{\left(x_{n}, y_{n}\right)}=\lim _{n \rightarrow \infty} A(\mathcal{F}, \varphi)\left(x_{n}, y_{n}\right)=A(\mathcal{F}, \varphi)(x, y)=\int \varphi d \mu_{(x, y)} .
$$

Since $\varphi \in \mathcal{C}(X \times X)$ was arbitrary, $\mu_{\left(x_{n}, y_{n}\right)}$ converges weakly to $\mu_{(x, y)}$ as $n \rightarrow \infty$ 
Regarding the opposite direction, observe that Theorem 2.2 gives that for each left $\mathrm{F} \varnothing$ lner sequence $\mathcal{F}$ and all $(x, y) \in X \times X$, we have

$$
D_{\mathcal{F}}(x, y)=\int d(z, w) d \mu_{(x, y)}(z, w) .
$$

Now, consider $\left(x_{n}, y_{n}\right) \in X \times X, n \in \mathbb{N}$ converging to $(x, y)$ as $n \rightarrow \infty$. Then

$$
\begin{aligned}
\lim _{n \rightarrow \infty} D\left(x_{n}, y_{n}\right) & =\lim _{n \rightarrow \infty} \sup \left\{D_{\mathcal{F}}\left(x_{n}, y_{n}\right): \mathcal{F} \text { is a left Følner sequence }\right\} \\
& =\lim _{n \rightarrow \infty} \int d(z, w) d \mu_{\left(x_{n}, y_{n}\right)}(z, w) \\
& =\int d(z, w) d \mu_{(x, y)}(z, w)=D(x, y) .
\end{aligned}
$$

Thus, $(X, G)$ is mean equicontinuous.

Let us conclude with a few comments on the natural question of why we have to formulate the assumptions of Theorem 4.2 (ii) for the product system. Obviously, a system is automatically pointwise uniquely ergodic if its product system has this property (if additionally $(x, y) \mapsto \mu_{(x, y)}$ is continuous, then $x \mapsto \mu_{x}$ is continuous as well). However, the converse is not true. For example, the product of a uniquely ergodic weakly mixing system with itself is ergodic with respect to the product measure. Hence, there are points whose orbit is dense in the full product and hence supports the product measure as well as the diagonal measure.

Furthermore, the next example shows that pointwise unique ergodicity of the product system (and hence, of the original system) and continuous dependence of the map $x \mapsto \mu_{x}$ does not imply continuity of the map $(x, y) \mapsto \mu_{(x, y)}$.

Example 4.3: Let $C \subseteq \mathbb{S}^{1}$ be a Cantor set which does not contain rationals ${ }^{1}$ and consider the skew-product $F: C \times \mathbb{S}^{1} \rightarrow C \times \mathbb{S}^{1}:(x, \theta) \mapsto(x, \theta+x)$. Clearly, the corresponding $\mathbb{Z}$-action is pointwise uniquely ergodic (the unique invariant measure supported on the orbit closure of $(x, \theta)$ is given by $\left.\mu_{(x, \theta)}=\delta_{x} \times m_{\mathbb{S}^{1}}\right)$ and the map $(x, \theta) \mapsto \mu_{(x, \theta)}$ is continuous. Furthermore, the product system is

\footnotetext{
${ }^{1}$ For example, one may take a sufficiently small cover $U$ of the rationals (e.g., a cover $U$ with $\left.m_{\mathbb{S} 1}(U)<1\right)$ and set $C=U^{c} \backslash \mathcal{I}$ where

$\mathcal{I}=\left\{x \in U^{c}:\right.$ there is $\varepsilon>0$ such that $B_{\varepsilon}(x) \cap U^{c}$ is at most countable $\}$.
} 
topologically conjugate to

$\hat{F}: C \times C \times \mathbb{S}^{1} \times \mathbb{S}^{1} \rightarrow C \times C \times \mathbb{S}^{1} \times \mathbb{S}^{1}:\left(x_{1}, x_{2}, \theta_{1}, \theta_{2}\right) \mapsto\left(x_{1}, x_{2}, \theta_{1}+x_{1}, \theta_{2}+x_{2}\right)$

and still pointwise uniquely ergodic. However, the map

$$
\left(x_{1}, x_{2}, \theta_{1}, \theta_{2}\right) \mapsto \mu_{\left(x_{1}, x_{2}, \theta_{1}, \theta_{2}\right)}
$$

cannot be continuous as this would imply mean equicontinuity (due to Theorem 4.2) while the MEF of $\left(C \times \mathbb{S}^{1}, F\right)$ coincides with the identity on $C$ so that the corresponding factor map is not a topo-isomorphy.

In fact, this can be seen explicitly: if $x_{1}, x_{2} \in C$ are rationally independent, then $\mu_{\left(x_{1}, x_{2}, \theta_{1}, \theta_{2}\right)}=\delta_{x_{1}} \times \delta_{x_{2}} \times m_{\mathbb{S}^{2}}$ (independently of $\theta_{1}$ and $\left.\theta_{2}\right)$. This is, of course, true for a dense set of points in $C \times C \times \mathbb{S}^{1} \times \mathbb{S}^{1}$. However, given any $x_{0} \in C$, we clearly have

$$
\mu_{\left(x_{0}, x_{0}, \theta_{1}, \theta_{2}\right)}=\delta_{x_{0}} \times \delta_{x_{0}} \times m_{\theta_{1}, \theta_{2}},
$$

where $m_{\theta_{1}, \theta_{2}}$ denotes the (one-dimensional) Lebesgue measure on the set

$$
\left\{\left(x+\theta_{1}, x+\theta_{2}\right): x \in \mathbb{S}^{1}\right\} \subseteq \mathbb{S}^{2} .
$$

Obviously, $\left(x_{1}, x_{2}, \theta_{1}, \theta_{2}\right) \mapsto \mu_{\left(x_{1}, x_{2}, \theta_{1}, \theta_{2}\right)}$ is not continuous in $\left(x_{0}, x_{0}, \theta_{1}, \theta_{2}\right)$ for $\theta_{1}, \theta_{2} \in \mathbb{S}^{1}$.

\section{Relating Besicovitch- and Weyl-mean equicontinuity}

By its very definition, Weyl-mean equicontinuity is a stronger assumption than Besicovitch- $\mathcal{F}$-mean equicontinuity. Quite remarkably, it turns out that Besicovitch- $\mathcal{F}$-mean equicontinuity, i.e., control over one Følner sequence $\mathcal{F}$, suffices to conclude Weyl-mean equicontinuity in many situations. A detailed study is given in this section and the presented results yield a proof of Theorem 1.3. By means of this result, we provide a non-trivial non-uniquely ergodic mean equicontinuous systems at the end of this section.

In the following, we will also speak of $\mathcal{F}$-mean equicontinuity with respect to a right $\mathrm{F} \varnothing$ lner sequence $\mathcal{F}$, where the definition is completely analogous to the definition using left Følner sequences given in (1).

Theorem 5.1: Let $(X, G)$ be $\mathcal{F}$-mean equicontinuous for some left Følner sequence $\mathcal{F}$ and let there be a $G$-invariant measure $\mu$ with $\operatorname{supp}(\mu)=X$. Then $(X, G)$ is mean equicontinuous. 
Remark 5.2: A comment on the assumption $\operatorname{supp}(\mu)=X$ may be in order. As is well known, every dynamical system $(X, G)$ possesses a $G$-invariant measure $\mu$ of maximal support, that is, a measure $\mu$ such that $\operatorname{supp}(\mu)$ contains the support of any other $G$-invariant measure. This support is clearly unique and coincides with the closure of the union of all supports of ergodic measures. While in general, $\operatorname{supp}(\mu)$ may not fill the whole space $X$, we can, of course, restrict attention to the maximal support and then apply the above theorem. By the Poicaré Recurrence Theorem, one may think of this as restricting to the recurrent dynamics of the system $(X, G)$.

Recall that for minimal dynamical systems every invariant measure has full support.

Corollary 5.3: If $(X, G)$ is minimal, then $\mathcal{F}$-mean equicontinuity for some left Følner sequence $\mathcal{F}$ implies mean equicontinuity.

Next we will collect some further assertions needed for the proof of Theorem 5.1. The following elementary lemma makes up for the (possible) lack of separability of $G$. Recall that $G$ is assumed to be $\sigma$-compact.

Lemma 5.4: Let $(X, G)$ be a dynamical system. Then there exists a countable subgroup $T \leq G$ such that $\overline{T x}=\overline{G x}$ for every $x \in X$.

Proof. Since $G$ is $\sigma$-compact, there exists an exhausting sequence $\left(K_{n}\right)_{n \in \mathbb{N}}$ of compact subsets of $G$. Given $\varepsilon>0$, set $T_{n} \subseteq K_{n}$ to be a finite subset such that for each $s \in K_{n}$ there is $t \in T_{n}$ with $\sup _{x \in X} d(s x, t x)<\varepsilon$. Note that $T_{n}$ is well defined due to the continuity of the defining action of $(X, G)$ as well as the compactness of $K_{n}$ and $X$. Set

$$
T^{\varepsilon}:=\bigcup_{n \in \mathbb{N}} T_{n}
$$

Then

$$
T^{\prime}:=\bigcup_{n \in \mathbb{N}} T^{1 / n}
$$

is countable and verifies $\overline{T^{\prime} x}=\overline{G x}$ for every $x \in X$. Letting $T$ be the group generated by $T^{\prime}$ proves the statement. 
Proposition 5.5: Suppose $(X, G)$ is $\mathcal{F}$-mean equicontinuous with respect to some left Følner sequence $\mathcal{F}$. Then the support of each ergodic measure $\mu$ is uniquely ergodic.

Proof. By possibly restricting to the support of $\mu$, we may assume without loss of generality that $X=\operatorname{supp}(\mu)$. By possibly going over to a subsequence of $\mathcal{F}$, we may further assume without loss of generality that there is a full measure set $X_{\mu}$ of $\mu$-generic points with respect to $\mathcal{F}$ (see Theorem 2.4).

From Proposition 4.1 we know that for each $\varphi \in \mathcal{C}(X)$ the maps $\bar{A}(\mathcal{F}, \varphi)(\cdot)$ and $\underline{A}(\mathcal{F}, \varphi)(\cdot)$ are continuous. Hence, with $T$ as in Lemma 5.4 and

$$
x_{0} \in \bigcap_{t \in T} t X_{\mu}
$$

we have that

$$
\bar{A}(\mathcal{F}, \varphi)(x)=\underline{A}(\mathcal{F}, \varphi)(x)=\mu(\varphi),
$$

for all $x$ from the set $T x_{0} \subseteq X_{\mu}$. Note that $T x_{0}$ is dense because of Lemma 5.4 and the fact that $\mu$-generic points are transitive. By the continuity of $\bar{A}(\mathcal{F}, \varphi)(\cdot)$ and $\underline{A}(\mathcal{F}, \varphi)(\cdot)$, we get that $A(\mathcal{F}, \varphi)(x)$, in fact, exists and coincides with $\mu(\varphi)$ for all $x \in X$. As $\varphi \in \mathcal{C}(X)$ was arbitrary, Theorem 2.2 yields the unique ergodicity of $(X, G)$.

Theorem 5.6: Suppose $(X, G)$ is $\mathcal{F}$-mean equicontinuous with respect to some left Følner sequence $\mathcal{F}$. Consider a point $x \in \operatorname{supp}(\mu)$ where $\mu$ is an arbitrary $G$-invariant measure. Then the orbit closure $\overline{G x}$ is uniquely ergodic.

Proof. By Theorem 2.2, it suffices to show that $A(\mathcal{F}, \varphi)(\cdot)$ exists and is constant on $\overline{G x}$ for each $\varphi \in \mathcal{C}(\overline{G x})$. In fact, by Tietze's Extension Theorem, it is enough to consider $\varphi \in \mathcal{C}(X)$. Observe that by Lemma 2.5 there is a sequence $\left(x_{n}\right)_{n \in \mathbb{N}}$ in $X$ with $x_{n} \rightarrow x$ for $n \rightarrow \infty$ such that each $x_{n}$ lies in the support of an ergodic measure. By Proposition 4.1, the functions $\bar{A}(\mathcal{F}, \varphi)(\cdot)$ and $\underline{A}(\mathcal{F}, \varphi)(\cdot)$, and hence $\bar{A}(\mathcal{F}, \varphi)(g \cdot)$ and $\underline{A}(\mathcal{F}, \varphi)(g \cdot)$, are continuous for every $g \in G$, so that

$$
\bar{A}(\mathcal{F}, \varphi)(g x)=\lim _{n \rightarrow \infty} \bar{A}(\mathcal{F}, \varphi)\left(g x_{n}\right)=\lim _{n \rightarrow \infty} \underline{A}(\mathcal{F}, \varphi)\left(g x_{n}\right)=\underline{A}(\mathcal{F}, \varphi)(g x),
$$

where we used the unique ergodicity on ergodic components (Proposition 5.5) in the second equality. This proves equality of $\bar{A}(\mathcal{F}, \varphi)(\cdot)$ and $\underline{A}(\mathcal{F}, \varphi)(\cdot)$ on $G x$. Similarly, we see that $\bar{A}(\mathcal{F}, \varphi)(\cdot)$ and $\underline{A}(\mathcal{F}, \varphi)(\cdot)$ are constant on $G x$. As both functions are continuous, this shows that $A(\mathcal{F}, \varphi)(\cdot)$ exists and is constant on $\overline{G x}$ for each $\varphi \in \mathcal{C}(X)$. 
Proof of Theorem 5.1. By Theorem 4.2, it suffices to show that $(X \times X, G)$ is pointwise uniquely ergodic and that the map $(x, y) \mapsto \mu_{(x, y)}$ is continuous. To that end, we first note that with $(X, G)$ the product system $(X \times X, G)$ is $\mathcal{F}$ mean equicontinuous as well. Moreover, by the assumptions, the measure $\mu \times \mu$ has full support on $X \times X$ which implies that $(X \times X, G)$ is pointwise uniquely ergodic, by Theorem 5.6.

It remains to show the continuity of the map $(x, y) \mapsto \mu_{(x, y)}$. By pointwise unique ergodicity and Theorem 2.2, we have for any $\varphi \in \mathcal{C}(X \times X)$ that

$$
\mu_{(x, y)}(\varphi)=\bar{A}(\mathcal{F}, \varphi)(x, y) .
$$

Hence, the continuity follows from Proposition 4.1 applied to $(X \times X, G)$.

In Theorem 5.1 we had to assume full support of the measure to deduce mean equicontinuity from $\mathcal{F}$-mean equicontinuity for some left Følner sequence $\mathcal{F}$. This is not needed if we know that a system is $\mathcal{F}$-mean equicontinuous for a right $\mathrm{F} \varnothing$ lner sequence $\mathcal{F}$. Details are discussed next.

Proposition 5.7: Let $\mathcal{F}=\left(F_{n}\right)_{n \in \mathbb{N}}$ be a right Følner sequence so that $(X, G)$ is $\mathcal{F}$-mean equicontinuous. If $(X, G)$ is transitive, then it has a unique $G$ invariant measure $\mu$ and there is a subsequence $\mathcal{F}^{\prime}=\left(F_{n}^{\prime}\right)_{n \in \mathbb{N}}$ of $\mathcal{F}$ such that

$$
\lim _{n \rightarrow \infty} \frac{1}{\left|F_{n}^{\prime}\right|} \int_{F_{n}^{\prime}} \varphi(t x) d m_{r}(t)=\mu(\varphi) \quad(x \in X),
$$

for each $\varphi \in \mathcal{C}(X)$.

Proof. Given $\varphi \in \mathcal{C}(X)$, we know by Proposition 4.1 that the maps $\bar{A}(\mathcal{F}, \varphi)(\cdot)$ and $\underline{A}(\mathcal{F}, \varphi)(\cdot)$ are continuous. Moreover, as $\mathcal{F}$ is a right Følner sequence, $\bar{A}(\mathcal{F}, \varphi)$ and $\underline{A}(\mathcal{F}, \varphi)$ are invariant and hence, due to the transitivity of $(X, G)$, constant.

Now, by the Stone-Weierstrass Theorem, $\mathcal{C}(X)$ is separable so that there exists a dense sequence of functions $\left(\varphi_{n}\right)_{n \in \mathbb{N}}$ in $\mathcal{C}(X)$. Observe that there is a subsequence $\mathcal{F}^{1}$ of $\mathcal{F}$ with $\bar{A}\left(\mathcal{F}^{1}, \varphi_{1}\right)=\underline{A}\left(\mathcal{F}^{1}, \varphi_{1}\right)$. Recursively, we obtain a subsequence $\mathcal{F}^{n+1}$ of $\mathcal{F}^{n}$ with

$$
\bar{A}\left(\mathcal{F}^{n+1}, \varphi_{n+1}\right)=\underline{A}\left(\mathcal{F}^{n+1}, \varphi_{n+1}\right)
$$

for each $n \in \mathbb{N}$. By setting $\mathcal{F}^{\prime}=\left(F_{n}^{n}\right)_{n \in \mathbb{N}}$, where $F_{n}^{n}$ is the $n$-th entry in $\mathcal{F}^{n}$, we eventually have a right $\mathrm{F} \varnothing$ lner sequence $\mathcal{F}^{\prime}$ with respect to which 
$\bar{A}\left(\mathcal{F}^{\prime}, \varphi_{n}\right)=\underline{A}\left(\mathcal{F}^{\prime}, \varphi_{n}\right)$ for all $n \in \mathbb{N}$. As $\bar{A}(\mathcal{F}, \varphi)$ and $\underline{A}(\mathcal{F}, \varphi)$ depend continuously on $\varphi$, we have

$$
\bar{A}\left(\mathcal{F}^{\prime}, \varphi\right)=\underline{A}\left(\mathcal{F}^{\prime}, \varphi\right)=\text { const },
$$

for all $\varphi \in \mathcal{C}(X)$. By using Proposition 2.3, we obtain the desired statement.

Theorem 5.8: If $(X, G)$ is $\mathcal{F}$-mean equicontinuous for some right Følner sequence $\mathcal{F}$, then $(X, G)$ is mean equicontinuous.

Proof. Given the preceding result, the proof is almost literally the same as that of Theorem 5.1.

Recall that a Følner sequence $\mathcal{F}$ is two-sided if it is a left and right $\mathrm{F} \varnothing$ lner sequence. If $G$ is unimodular, there always exists a two-sided Følner sequence; see [Eme74, Theorem 1"]. In particular (and trivially), if $G$ is abelian, every Følner sequence is two-sided. We immediately obtain the following corollaries.

Corollary 5.9: Suppose $G$ is unimodular and let $(X, G)$ be $\mathcal{F}$-mean equicontinuous for a two-sided $F \varnothing l n e r$ sequence $\mathcal{F}$. Then $(X, G)$ is mean equicontinuous.

Corollary 5.10: If $G$ is abelian and $(X, G)$ is $\mathcal{F}$-mean equicontinuous for some Følner sequence $\mathcal{F}$, then $(X, G)$ is mean equicontinuous.

Proof of Theorem 1.3. This theorem is now an immediate consequence of Theorem 5.1 and Corollary 5.10.

Last, we would like to address the question of whether there are non-trivial non-uniquely ergodic mean equicontinuous systems (that is, non-uniquely ergodic mean equicontinuous systems which are neither finite unions of uniquely ergodic systems nor products of such). The following example demonstrates that such non-trivial neither minimal nor uniquely ergodic systems exist.

Example 5.11: Given a sequence $x=\left(x_{k}\right)_{k \in \mathbb{Z}} \in\{0,1\}^{\mathbb{Z}}$ and $p \in \mathbb{N}$, let us set the $p$-periodic part of $x$ to be

$$
\operatorname{Per}(x, p):=\left\{k \in \mathbb{Z} \mid x_{k}=x_{k+n p}(n \in \mathbb{Z})\right\} .
$$

We put $\mathcal{T}$ to be the closure of

$$
\mathcal{T}^{\prime}:=\left\{x \in\{0,1\}^{\mathbb{Z}} \mid \emptyset \neq \operatorname{Per}\left(x, 2^{n}\right) \subsetneq \operatorname{Per}\left(x, 2^{n+1}\right)(n \in \mathbb{N})\right\}
$$

in $\{0,1\}^{\mathbb{Z}}$ (equipped with the product topology). Observe that for every $x \in \mathcal{T}^{\prime}$ and each $n \in \mathbb{N}$, we have that there is exactly one $k \in\left[0,2^{n}-1\right] \backslash \operatorname{Per}\left(x, 2^{n}\right)$. 
Clearly, $\mathcal{T}$ is $\sigma$-invariant where $\sigma:\{0,1\}^{\mathbb{Z}} \rightarrow\{0,1\}^{\mathbb{Z}}$ denotes the left shift. We show that $(\mathcal{T}, \sigma)$ is mean equicontinuous by proving that it is $\mathcal{F}$-mean equicontinuous for $\mathcal{F}=\left(\left[0,2^{n}-1\right]\right)_{n \in \mathbb{N}}$; see Corollary 5.10. To that end, define $D^{n}$ to be the pseudometric given by

$$
D^{n}(x, y):=1 / 2^{n} \cdot \sum_{\ell=0}^{2^{n}-1} d\left(\sigma^{\ell}(x), \sigma^{\ell}(y)\right),
$$

where we consider $d$ to be the Cantor metric with

$$
d(x, y):=2^{-\min \left\{|k| \mid k \in \mathbb{Z} \text { and } x_{k} \neq y_{k}\right\}} .
$$

By definition,

$$
\limsup _{n \rightarrow \infty} D^{n}(x, y)=D_{\mathcal{F}}(x, y)
$$

for $x, y \in\{0,1\}^{\mathbb{Z}}$.

Now, given $x_{1}, x_{2} \in \mathcal{T}^{\prime}$ with $d\left(x_{1}, x_{2}\right) \leq 2^{-2^{n}}$, observe that there are at most two elements in $\left[0,2^{n}-1\right] \backslash\left(\operatorname{Per}\left(x_{1}, 2^{n}\right) \cap \operatorname{Per}\left(x_{2}, 2^{n}\right)\right)$ so that

$$
\begin{aligned}
D^{k}\left(x_{1}, x_{2}\right) & =1 / 2^{k-n} \cdot \sum_{m=0}^{2^{k-n}-1} D^{n}\left(\sigma^{m \cdot 2^{n}}\left(x_{1}\right), \sigma^{m \cdot 2^{n}}\left(x_{2}\right)\right) \\
& \leq \max _{m=0, \ldots, 2^{k-n}-1} D^{n}\left(\sigma^{m \cdot 2^{n}}\left(x_{1}\right), \sigma^{m \cdot 2^{n}}\left(x_{2}\right)\right) \\
& \leq 1 / 2^{n} \cdot 4 \cdot \sum_{\ell=0}^{\infty} 2^{-\ell}=2^{-n+3},
\end{aligned}
$$

for all $k \geq n$. Now, given $y \in \mathcal{T}$, let $\left(x_{n}\right)_{n \in \mathbb{N}}$ be a sequence in $\mathcal{T}^{\prime}$ with $d\left(x_{n}, y\right) \leq 2^{-2^{n}}$. Observe that

$$
D^{n}\left(x_{n}, y\right) \leq 1 / 2^{n} \cdot \sum_{\ell=1}^{2^{n}} 2^{-\ell} \leq 2^{-n}
$$

as well as $d\left(x_{n}, x_{k}\right) \leq 2^{-2^{n}}$ for $k \geq n$. Hence,

$$
D^{k}\left(x_{n}, y\right) \leq D^{k}\left(x_{n}, x_{k}\right)+D^{k}\left(x_{k}, y\right) \leq 2^{-n+3}+2^{-k} \text { for all } k \geq n
$$

so that $D_{\mathcal{F}}\left(x_{n}, y\right) \leq 2^{-n+3}$. This yields the $\mathcal{F}$-mean equicontinuity of $(\mathcal{T}, \sigma)$. Observe that $\mathcal{T}$ contains a dense set of points which are periodic with respect to $\sigma$ as well as a dense set of infinite (i.e., non-periodic) subshifts (in fact, regular Toeplitz subshifts). 


\section{Mean equicontinuity and discrete spectrum}

In this section, we establish a relation between mean equicontinuity and discrete spectrum, see also [GR17, $\mathrm{HLT}^{+} 21, \mathrm{YZZ19]}$ for related discussions. A dynamical system $(X, G)$ together with an invariant measure $\mu$ is said to have discrete spectrum if $L_{2}(X, \mu)$ can be written as an orthogonal sum of finitedimensional, $G$-invariant subspaces $V_{\alpha}$, where $\alpha$ runs through some index set; see [Mac64] for further details. As before, we will denote by $(\mathbb{T}, G)$ the maximal equicontinuous factor of $(X, G)$ and by $\pi: X \rightarrow \mathbb{T}$ a corresponding factor map.

We will need the following well-known fact which follows from the general theory of Ellis semigroups of equicontinuous systems (see, for example, [Aus88, pp. 52-53]): if $\left(\mathbb{T}^{\prime}, G\right)$ is minimal and equicontinuous, then $\mathbb{T}^{\prime}$ is homeomorphic to a homogeneous space, that is, there is a compact group $E\left(\mathbb{T}^{\prime}\right)$ and a closed subgroup $F \leq E\left(\mathbb{T}^{\prime}\right)$ (in general not normal) such that $\mathbb{T}^{\prime}$ is homeomorphic to the set of left cosets $E\left(\mathbb{T}^{\prime}\right) / F$. If $G$ is abelian, then $E\left(\mathbb{T}^{\prime}\right)$ is abelian and $\mathbb{T}^{\prime}$ is homeomorphic to $E\left(\mathbb{T}^{\prime}\right)$.

Theorem 6.1: Suppose $(X, G)$ is minimal. Then, the following assertions are equivalent:

(i) The system $(X, G)$ is mean equicontinuous.

(ii) $(X, G)$ is uniquely ergodic and, if $\mu$ denotes the unique invariant probability measure, then $L_{2}(X, \mu)$ can be written as an orthogonal sum of finite dimensional, $G$-invariant subspaces $V_{\alpha}$, consisting of continuous functions ( $\alpha$ runs through some index set $I$ ).

Proof. (i) $\Rightarrow$ (ii): Several results of the previous sections imply that every minimal mean equicontinuous system is uniquely ergodic. Let us hence denote by $\mu$ the unique $G$-invariant measure on $X$. Now, observe that if $L_{2}(\mathbb{T}, \pi(\mu))$ can be decomposed as an orthogonal sum of finite-dimensional $G$-invariant subspaces consisting of continuous functions, then this holds true for $L_{2}(X, \mu)$ as well. This follows from the unitarity of $U_{\mu}$ (defined as in (2); see also Theorem 3.7 and Proposition 2.1) and the fact that $U_{\mu}$ maps continuous functions to continuous functions (due to the continuity of $\pi$ ).

Therefore, it suffices to find a corresponding decomposition of $L_{2}(\mathbb{T}, \pi(\mu))$. If $\mathbb{T}$ is homeomorphic to the compact group $E(\mathbb{T})$ from above, this decomposition is provided by the classical Peter-Weyl Theorem. In case that $\mathbb{T}$ is homeomorphic to a homogeneous space, the decomposition is obtained by a standard extension of the Peter-Weyl Theorem to homogeneous spaces. 
(ii) $\Rightarrow\left(\right.$ i): For each $\alpha \in I$ we define the pseudometric $d_{\alpha}$ on $X$ via

$$
d_{\alpha}(x, y):=\sup \left\{|f(x)-f(y)| \mid f \in V_{\alpha},\|f\|_{\infty}=1\right\} .
$$

As $V_{\alpha}$ is finite-dimensional and consists of continuous functions, each $d_{\alpha}$ is continuous. As $V_{\alpha}$ is $G$-invariant, each $d_{\alpha}$ is $G$-invariant. Further, observe that the separability of $L_{2}(X, \mu)$ implies that $I$ is countable. Thus, we may consider the pseudometric

$$
D^{\prime}=\sum_{\alpha} c_{\alpha} \cdot d_{\alpha}
$$

where $\left(c_{\alpha}\right)_{\alpha \in I}$ is some summable sequence of positive numbers.

We can hence introduce an invariant and closed equivalence relation on $X$ by

$$
x \sim y: \Longleftrightarrow d_{\alpha}(x, y)=0 \text { for all } \alpha \in I \quad\left(\Longleftrightarrow D^{\prime}(x, y)=0\right) .
$$

Then

$$
Y:=X / \sim
$$

is a compact space which we may consider equipped with the metric $D^{\prime}$ in the obvious way. Further, $(Y, G)$ (where the action of $G$ on $Y$ is defined in the canonical way) is an isometric and hence equicontinuous factor, as $D^{\prime}$ is $G$-invariant. Let $h: X \rightarrow Y$ be the factor map and note that $V: L^{2}(Y, h(\mu)) \rightarrow L^{2}(X, \mu)$ with

$$
V f=f \circ h
$$

is unitary (as we only identify points which can not be distinguished by elements of the $V_{\alpha}$ ). Now, the application of Theorem 3.7 yields (i).

Remark 6.2:

(a) The assumption of minimality of $(X, G)$ can be slightly weakened to unique ergodicity or transitivity, where the latter implies the former due to Theorem 3.4. In fact, for (ii) $\Rightarrow$ (i) we did not need the minimality of $(X, G)$. For (i) $\Rightarrow($ ii) note that $(\mathbb{T}, G)$ is still minimal (see Proposition 3.3).

(b) The Peter-Weyl Theorem used in the proof of (i) $\Rightarrow$ (ii) actually gives one more feature of the finite-dimensional subspaces appearing in (ii). They can be assumed to be irreducible. Here, a $G$-invariant subspace $V$ of $L_{2}(X, \mu)$ is called irreducible if it can not be written as an orthogonal sum of two non-trivial $G$-invariant subspaces. 
In the case of abelian $G$, we obtain a somewhat stronger statement. As this is of interest in various contexts, we include a discussion.

Corollary 6.3: Let $G$ be abelian. Suppose $(X, G)$ is minimal. Then, the following assertions are equivalent:

(i) The system $(X, G)$ is mean equicontinuous.

(ii) The system $(X, G)$ is uniquely ergodic and, if $\mu$ denotes the unique invariant probability measure, then $L^{2}(X, \mu)$ has an orthonormal basis of continuous eigenfunctions.

Proof. Clearly, condition (ii) of the present corollary is stronger than condition (ii) of the previous theorem. Thus, it suffices to show (i) $\Rightarrow$ (ii). This can be seen as in the proof of the previous theorem after noting that $\mathbb{T}$ is homeomorphic to the compact group $E(\mathbb{T})$. With this in mind, statement (ii) is a direct consequence of the duality theory for compact abelian groups. Alternatively, one may also argue that the irreducible subspaces appearing in Theorem 6.1(ii) must be one-dimensional in the abelian case.

Remark 6.4: The last three decades have seen tremendous interest in the field of aperiodic order, also known as mathematical quasicrystals (see [BG13, KLS15] for extensive discussions). The common way to model aperiodic order is via dynamical systems over the group $\mathbb{R}^{n}$. In typical examples, these systems will be uniquely ergodic and minimal. In any case, such a system comes with a diffraction measure. As mentioned in the introduction, a key effort is to show that the diffraction measure is a pure point measure. This in turn has been proven to be equivalent to discrete spectrum of the underlying dynamical system. Hence, discrete spectrum is at the core of aperiodic order. In the further analysis of the diffraction measure, continuity of the eigenfunctions turns out to play a role. Indeed, it is exactly under this condition that a convincing positive answer to the so-called Bombieri-Tayler Conjecture can be given [Len09] (see [Rob99] for related earlier results as well.) Given this situation, the class of minimal uniquely ergodic systems with discrete spectrum and continuous eigenfunctions (which is characterized in the preceding corollary) presents itself as a very natural candidate for models of aperiodic order. 


\section{Non-abelian examples and conclusions}

7.1. IsOMETRIC SUBGROUPS OF TOPOLOGICAL FULL GROUPS. In this section, we provide means to construct new examples of (in particular, non-abelian) mean equicontinuous group actions by using suitable subgroups of the topological full group of known mean equicontinuous systems. Recall that the topological full group $\llbracket(Z, G) \rrbracket$ of a dynamical system $(Z, G)$ is the group of all homeomorphisms on $Z$ which locally coincide with an element of $G$ equipped with the uniform topology. For simplicity, let us restrict to systems where $Z$ is a Cantor space. In this case, a homeomorphism $s: Z \rightarrow Z$ is an element of $\llbracket(Z, G) \rrbracket$ if and only if for every $z_{0} \in Z$ there is a clopen neighborhood $U$ of $z_{0}$ and an element $g \in G$ such that $s z=g z$ for all $z \in U$.

We make use of the following structural result. Recall that a group $G$ acts freely on $Z$ if $g z=z$ for some $z \in Z$ and $g \in G$ implies that $g$ is the identity.

Theorem 7.1 ([CM16, Corollary 4.9]): Suppose $Z$ is a Cantor space and $G$ is countable. Further, assume $G$ acts minimally, equicontinuously and freely on $Z$. Then the topological full group $\llbracket(Z, G) \rrbracket$ is amenable if and only if $G$ is amenable.

Suppose we are in the situation of the previous statement, in particular, $G$ acts equicontinuously on $(Z, d)$. Without loss of generality we may assume that $G$ acts isometrically with respect to $d$ (see Section 1.1). We define the isometric subgroup $\llbracket(Z, G) \rrbracket_{I} \leq \llbracket(Z, G) \rrbracket$ to be that subgroup which comprises all elements of $\llbracket(Z, G) \rrbracket$ that act isometrically on $Z$ with respect to $d$. Clearly, $\llbracket(Z, G) \rrbracket_{I}$ is a closed subgroup of $\llbracket(Z, G) \rrbracket$ and hence amenable due to Theorem 7.1.

Now, if $(X, G)$ is an extension of $(Z, G)$ via the factor map $h: X \rightarrow Z$ with $Z$ a Cantor space, then $\llbracket(Z, G) \rrbracket_{I}$ acts naturally on $X$ : given $s \in \llbracket(Z, G) \rrbracket_{I}$ with a (finite) clopen partition $\left\{Z_{i}\right\}$ of $Z$ and elements $\left\{g_{i}\right\} \subseteq G$ such that $\left.s\right|_{Z_{i}}=\left.g_{i}\right|_{Z_{i}}$, then let $s x=g_{i} x$ whenever $x \in h^{-1}\left(Z_{i}\right)$. Furthermore, we immediately see that $h$ still is a factor map from $\left(X, \llbracket(Z, G) \rrbracket_{I}\right)$ to $\left(Z, \llbracket(Z, G) \rrbracket_{I}\right)$.

Theorem 7.2: Suppose $(X, G)$ is mean equicontinuous and uniquely ergodic with a maximal equicontinuous factor $(\mathbb{T}, G)$ where $\mathbb{T}$ is a Cantor space. If $G$ is countable and acts freely on $\mathbb{T}$, then we have that $\left(X, \llbracket(\mathbb{T}, G) \rrbracket_{I}\right)$ is mean equicontinuous with $M E F\left(\mathbb{T}, \llbracket(\mathbb{T}, G) \rrbracket_{I}\right)$. 
Proof. Since $(X, G)$ is uniquely ergodic, Proposition 3.3 yields that $(\mathbb{T}, G)$ is minimal. Hence, $(\mathbb{T}, G)$ verifies the assumptions of Theorem 7.1. Clearly, every $\llbracket(\mathbb{T}, G) \rrbracket_{I}$-invariant measure on $X$ (on $\mathbb{T}$ ) necessarily is also a $G$-invariant measure on $X$ (on $\mathbb{T})$. Therefore, $\left(X, \llbracket(\mathbb{T}, G) \rrbracket_{I}\right)$ is a topo-isomorphic extension of the equicontinuous system $\left(\mathbb{T}, \llbracket(\mathbb{T}, G) \rrbracket_{I}\right)$. By Theorem 3.7, the statement follows.

Last, we present a straightforward instructive application of Theorem 7.2. Let us point out that all the considerations in the following example directly generalize to higher-dimensional odometers and associated regular Toeplitz configurations; see [Cor06].

Example 7.3: We assume that the reader is familiar with the theory of odometers/adding machines; see, for instance, [Ků03, Dow05] for further information. We consider the dyadic odometer $\left(\mathbf{2}^{\mathbb{N}}, \mathbb{Z}\right)$. That is, $\mathbf{2}^{\mathbb{N}}$ is the compact group obtained as the inverse limit

$$
\mathbf{2}^{\mathbb{N}}:=\lim _{\ell \in \mathbb{N}} \mathbb{Z} / 2^{\ell} \mathbb{Z}
$$

and $n \in \mathbb{Z}$ acts on $\theta \in \mathbf{2}^{\mathbb{N}}$ by

$$
\theta \mapsto \theta+n,
$$

where we consider $n$ as an element of $\mathbf{2}^{\mathbb{N}}$.

Now, the isometric subgroup $\llbracket\left(2^{\mathbb{N}}, \mathbb{Z}\right) \rrbracket_{I}$ contains, among others, the element $s$ given by

$$
s \theta=s\left(\theta_{0}, \theta_{1}, \ldots\right):= \begin{cases}\theta & \text { if } \theta_{0}=0 \\ \theta+2 & \text { if } \theta_{0}=1 .\end{cases}
$$

Obviously,

$$
s(\theta+1) \neq 1+s \theta .
$$

Hence, $\llbracket\left(2^{\mathbb{N}}, \mathbb{Z}\right) \rrbracket_{I}$ is a non-abelian amenable group which acts mean equicontinuously (according to Theorem 7.2) on, in particular, the shift orbit closure of any regular Toeplitz sequence whose MEF is given by $\left(2^{\mathbb{N}}, \mathbb{Z}\right.$ ) (for concrete examples, see also [Ko03, Dow05]). Obviously, these orbit closures are Cantor spaces as well. To obtain examples where the domain is not totally disconnected, we can consider Auslander systems, see [HJ97], which also have odometers as their $\mathrm{MEF}$ and are mean equicontinuous. 
7.2. Irregular extensions. Suppose $(X, G)$ is an extension of $(Y, G)$ via the factor map $h: X \rightarrow Y$. We say $(X, G)$ is a regular extension of $(Y, G)$ if for every $G$-invariant measure $\mu$ on $X$ we have that

$$
h(\mu)\left(\left\{y \in h(X): \# h^{-1}(y)>1\right\}\right)=0 ;
$$

otherwise we say $(X, G)$ is an irregular extension. Given $y \in Y$, we refer to $h^{-1}(y)$ as its fiber.

Note that a regular extension is automatically a topo-isomorphic extension (see also [GJY21] for a recent and in-depth discussion of the relation between regularity and mean equicontinuity of $\mathbb{Z}_{+}$-actions). Examples of regular extensions of equicontinuous systems are Sturmian subshifts, regular Toeplitz subshifts and the Denjoy systems described in Example 3.22. There are also irregular topo-isomorphic extensions of equicontinuous systems. The Cantor substitution subshift in Example 3.21 is a transitive irregular extension of the trivial system. Minimal examples can be found in [DK15, DG16, FGJO18], where [DK15, Example 5.1] and the example in [FGJO18, Section 5.4] have almost surely (with respect to the unique invariant measure of their MEFs) countable fibers but still a residual set of points whose fibers are singletons. In contrast, in the examples constructed in [DG16, Section 3], every fiber is uncountable. Indeed, in this subsection, we will show that almost every fiber of an irregular extension must be at least countable. For the convenience of the reader, we provide a proof of the next statement.

Lemma 7.4: Let $(X, G)$ be an extension of $(Y, G)$ via the factor map $h: X \rightarrow Y$ and let $\mu$ be an ergodic $G$-invariant measure on $Y$. Suppose $h^{-1}(y)$ is finite for $\mu$-almost every $y \in Y$. Then there is $n_{0} \in \mathbb{N}$ such that $\mu$-almost everywhere we have $\# h^{-1}=n_{0}$.

Proof. Observe that $h$ gives rise to an upper semi-continuous and hence Borel measurable map $\gamma$ from $Y$ to the space of compact subsets of $X$ (endowed with the Hausdorff metric), defined by $\gamma(y)=\pi^{-1}(y)$ for each $y \in Y$. By Lusin's Theorem, there is a compact set $K^{\prime} \subseteq Y$ with $\mu\left(K^{\prime}\right)>0$ such that $\left.\gamma\right|_{K^{\prime}}$ is continuous. Set

$$
Y^{\prime}:=\left\{y \in Y: \# h^{-1}(y)<\infty\right\} .
$$

By the assumptions, $\mu\left(Y^{\prime}\right)=1$. Since $\mu$ is an inner regular measure, we may assume w.l.o.g. that $K^{\prime} \subseteq Y^{\prime}$. Let $K \subseteq K^{\prime}$ be the support of the measure $\left.\mu\right|_{K^{\prime}}$. Clearly, $\mu(K)>0$. 
Pick some $y_{0} \in K$ and set $n_{0}:=\# h^{-1}\left(y_{0}\right)$. By continuity of $\gamma$ on $K$, there is $\delta>0$ such that for all $y \in B_{\delta}\left(y_{0}\right) \cap K$ we have

$$
d\left(\gamma(y), \gamma\left(y_{0}\right)\right)<\frac{1}{2} \cdot \min _{x_{1} \neq x_{2} \in h^{-1}\left(y_{0}\right)} d\left(x_{1}, x_{2}\right)
$$

and hence $\# h^{-1}(y) \geq n_{0}$. Since $G$ acts on $X$ by homeomorphisms, we actually have $\# h^{-1}(y) \geq n_{0}$ for each $y$ in the invariant set

$$
A=\bigcup_{g \in G} g\left(B_{\delta}\left(y_{0}\right) \cap K\right) .
$$

By definition of $K, \mu\left(B_{\delta}\left(y_{0}\right) \cap K\right)>0$ so that $A$ is of full measure, since $\mu$ is ergodic.

Set

$$
\mathcal{N}(K):=\left\{n \in \mathbb{N} \text { : there is } y \in K \text { such that } \# h^{-1}(y)=n\right\} .
$$

If we can show that $\mathcal{N}(K)$ is bounded, the above proves the statement. Assume for a contradiction that $\mathcal{N}(K)$ is unbounded. The above shows: for all $n \in \mathcal{N}(K)$, we have $\mu\left(\left\{y \in Y: \# h^{-1}(y) \geq n\right\}\right)=1$ or, in other words,

$$
\mu\left(\left\{y \in Y: \# h^{-1}(y)<n\right\}\right)=0 .
$$

Hence, $\mu\left(Y^{\prime}\right)=\mu\left(\bigcup_{n \in \mathcal{N}(K)}\left\{y \in Y: \# h^{-1}(y)<n\right\}\right)=0$ which is an obvious contradiction.

Theorem 7.5: Let $(X, G)$ be an irregular extension of $(Y, G)$ via the factor map $h: X \rightarrow Y$, that is,

$$
h(\mu)\left(\left\{y \in h(X): \# h^{-1}(y)>1\right\}\right)>0
$$

for some $G$-invariant measure $\mu$ on $X$. If $h(\mu)$-almost all fibers of $h$ are finite, then $h$ is not a topo-isomorphy.

Proof. We may assume without loss of generality that $\mu$ is ergodic, because of Lemma 2.5. For a contradiction, assume that $(X, G)$ is a topo-isomorphic extension of $(Y, G)$ via $h$.

By definition of topo-isomorphy, there is a Borel measurable map $\gamma: Y \rightarrow X$ such that for all $g \in G$ we have $\gamma(g y)=g \gamma(y)$ for $h(\mu)$-almost all $y$ and

$$
\int \varphi d \mu=\int \varphi \circ \gamma d h(\mu)
$$


for every $\varphi \in \mathcal{C}(X)$. By Lemma 7.4, we further have that $h(\mu)$-almost all fibers are of equal cardinality $n_{0}>1$. By the Riesz Representation Theorem, we can define a probability measure $\nu$ on $X$ by

$$
\varphi \longmapsto \int_{Y} \frac{1}{n_{0}-1} \cdot \sum_{\substack{x \in h^{-1}(y) \\ x \neq \gamma(y)}} \varphi(x) d h(\mu)(y) \quad(\varphi \in \mathcal{C}(X)) .
$$

Observe that $\nu$ is $G$-invariant and $\nu \neq \mu$. Moreover, $h(\nu)=h(\mu)$ and this implies $\nu$ is ergodic since $h$ is assumed to be a topo-isomorphy. However, this yields a contradiction, according to Proposition 3.1.

We immediately obtain the next two statements, using Corollary 3.20 for the second one.

Corollary 7.6: Assume $(X, G)$ has a unique $G$-invariant measure $\mu$ and is an irregular topo-isomorphic extension of a system $(Y, G)$ via a factor map $h: X \rightarrow Y$. Then for $h(\mu)$-almost every $y \in Y$ we have that $h^{-1}(y)$ is infinite.

Corollary 7.7: Suppose that $(X, G)$ is an irregular extension of $(Y, G)$ via the factor map $h: X \rightarrow Y$ and suppose the MEF of $(X, G)$ and $(Y, G)$ coincide. If the fibers of $h$ are finite, then $(X, G)$ can not be mean equicontinuous.

An example fitting into the setting of the second corollary is the Thue-Morse subshift which is a 2-1 extension of a regular Toeplitz subshift with the same maximal equicontinuous factor (see, for instance, [BG13] for more information). In particular, we get that the Thue-Morse system is not mean equicontinuous.

7.3. Maximally almost PeRIOdic GRoups. In this last section we show that if a group $G$ acts minimally, mean equicontinuously and effectively on a compact metric space $(X, d)$, then it is necessarily maximally almost periodic.

Recall that $G$ acts effectively on $X$ if for each $g \in G$ which is different from the neutral element $e \in G$, there is $x \in X$ with $g x \neq x$. Recall further that a topological group $G$ is maximally almost periodic (MAP) if $G$ admits a continuous and injective homomorphism into a compact Hausdorff group; see, for instance, [vN34]. Note that a locally compact MAP group is necessarily unimodular [LR68]. We will make use of the following characterization of maximal almost periodicity [Hua79]: a topological group $G$ is MAP if and only if $G$ admits an equicontinuous and effective action on a compact Hausdorff space. 
Theorem 7.8: Let $(X, G)$ be a dynamical system and denote by $(\mathbb{T}, G)$ its maximal equicontinuous factor. If $(X, G)$ is mean equicontinuous, allows for an invariant measure of full support and $G$ acts effectively on $X$, then $G$ also acts effectively on $\mathbb{T}$. In particular, this implies that $G$ is maximal almost periodic and unimodular.

Proof. As before, we denote by $\pi$ a factor map from $X$ to $\mathbb{T}$. Let $\mu$ be an invariant measure with full support. Since $\pi$ is a topo-isomorphy, there are subsets $M \subseteq X$ and $N \subseteq \mathbb{T}$ of full $\mu$ - and $\pi(\mu)$-measure, respectively, such that the restriction of $\pi$ to $M$ is a bijection from $M$ onto $\pi(M)=N$.

Now, assume there is $g \in G$ with $g y=y$ for all $y \in \mathbb{T}$. Observe that such $g$ has to verify $g x=x$ for $\mu$-almost all $x \in M$, since $\pi$ restricted to $M$ is injective and since -by the invariance of $\mu$-almost every point of $M$ is mapped into $M$ under the action of $g$. As $\mu$ is of full support, every full-measure set is dense in $X$. Thus, the continuity of $g$ implies $g x=x$ for all $x \in X$. As $G$ acts effectively on $X$, this gives $g=e$.

Recall that for a minimal dynamical system all measures have full support.

Corollary 7.9: If $G$ acts minimally, mean equicontinuously and effectively on $X$, then $G$ is maximal almost periodic and unimodular.

We would like to close with a partial answer to the following question [GM18b, Question 18.60]: which discrete countable groups $G$ have effective tame minimal actions? Here, the term tame refers to a certain low dynamical complexity of a dynamical system (see, e.g., [Gla18]). Now, according to [Gla18, Corollary $5.4(2)]$, if $(X, G)$ is tame and $G$ amenable, then $(X, G)$ is a topo-isomorphic extension of its MEF and hence mean equicontinuous, due to Theorem 3.7 (for $\mathbb{Z}$-actions, see also [Gla18, Corollary 5.10]). Thus, from Theorem 7.8 we obtain that among the amenable, discrete countable groups exactly the maximally almost periodic ones allow for an effective tame minimal action.

\section{References}

[AB98] P. Alessandri and V. Berthé, Three distance theorems and combinatorics on words, L'Enseignement Mathématique 44 (1998), 103-132.

[Aus59] J. Auslander, Mean-L-stable systems, Illinois Journal of Mathematics 3 (1959), $566-579$. 
[Aus88] J. Auslander, Minimal Flows and their Extensions, North-Holland Mathematics Studies, Vol. 153, North-Holland, Amsterdam, 1988.

[BG13] M. Baake and U. Grimm, Aperiodic Order, Encyclopedia of Mathematics and its Applications, Vol. 149, Cambridge University Press, Cambridge, 2013.

[BL17] M. Baake and D. Lenz, Spectral notions of aperiodic order, Discrete \& Continuous Dynamical Systems. Series S 10 (2017), 161-190.

[CM16] M. I. Cortez and K. Medynets, Orbit equivalence rigidity of equicontinuous systems, Journal of the London Mathematical Society 94 (2016), 545-556.

[Cor06] M. I. Cortez, $\mathbb{Z}^{d}$ Toeplitz arrays, Discrete \& Continuous Dynamical Systems 15 (2006), 859-881.

[DD02] T. Downarowicz and F. Durand, Factors of Toeplitz flows and other almost 1-1 extensions over group rotations, Mathematica Scandinavica 90 (2002), 57-72.

[Den32] A. Denjoy, Sur les courbes définies par les équations différentielles à la surface du tore, Journal de Mathématiques Pures et Appliquées 11 (1932), 333-376.

[DG16] T. Downarowicz and E. Glasner, Isomorphic Extensions and Applications, Topological Methods in Nonlinear Analysis 48 (2016), 321-338.

[DI88] T. Downarowicz and A. Iwanik, Quasi-uniform convergence in compact dynamical systems, Studia Mathematica 89 (1988), 11-25.

[DK15] T. Downarowicz and S. Kasjan, Odometers and Toeplitz systems revisited in the context of Sarnak's conjecture, Studia Mathematica 229 (2015), 45-72.

[Dow05] T. Downarowicz, Survey of odometers and Toeplitz flows, in Algebraic and Topological Dynamics, Contemporary Mathe,atics, Vol. 385, American Mathematical Society, Providence, RI, 2005, pp. 7-37.

[DT97] M. Drmota and R. F. Tichy, Sequences, Discrepancies and Applications. Lecture Notes in Mathematics, Vol. 1651, Springer, Berlin, 1997.

[Eme74] W. R. Emerson, Large symmetric sets in amenable groups and the individual ergodic theorem, American Journal of Mathematics 96 (1974), 242-247.

[EW11] M. Einsiedler and T. Ward, Ergodic Theory with a View towards Number Theory, Graduate Texts in Mathematics, Vol. 259, Springer, London, 2011.

[Far62] R. H. Farrell, Representation of invariant measures, Illinois Journal of Mathematics 6 (1962), 447-467.

[FGJO18] G. Fuhrmann, E. Glasner, T. Jäger and C. Oertel, Irregular model sets and tame dynamics, Transactins of the American Mathematical Society 374 (2021), 37033734 .

[FK02] R. Feres and A. Katok, Ergodic theory and dynamics of G-spaces (with special emphasis on rigidity phenomena), in Handbook of Dynamical Systems. Vol. 1A, North-Holland, Amsterdam, 2002, pp. 665-763.

[FK20] G. Fuhrmann and D. Kwietniak, On tameness of almost automorphic dynamical systems for general groups, Bulletin of the London Mathematical Society $\mathbf{5 2}$ (2020), 24-42.

[Fog02] N. P. Fogg, Substitutions in Dynamics, Arithmetics and Combinatorics, Lecture Notes in Mathematics, Vol. 1794, Springer, Berlin-Heidelberg, 2002.

[Fom51] S. Fomin, On dynamical systems with a purely point spectrum, Doklady Akademii Nauk SSSR 77 (1951), 29-32. 
[Fur81] H. Furstenberg, Recurrence in Ergodic Theory and Combinatorial Number Theory, Princeton University Press, Princeton, NJ, 1981.

[GJY21] F. García-Ramos, T. Jäger and X. Ye, Mean equicontinuity, almost automorphy and regularity, Israel Journal of Mathematics 243 (2021), 155-183.

[GL20] M. Gröger and O. Lukina, Measures and stabilizers of group Cantor actions, Discrete \& Continuous Dynamical Systems 41 (2021), 2001-2029.

[Gla18] E. Glasner, The structure of tame minimal dynamical systems for general groups, Inventiones mathematicae 211 (2018), 213-244.

[GM18a] E. Glasner and M. Megrelishvili, Circularly ordered dynamical systems, Monatshefte für Mathematik 185 (2018), 415-441.

[GM18b] E. Glasner and M. Megrelishvili, More on tame dynamical systems, in Ergodic Theory and Dynamical Systems in their Interactions with Arithmetics and Combinatorics, Lecture Notes in Mathematics, Vol. 2213, Springer, Cham, 2018, pp. 351392.

[GR17] F. García-Ramos, Weak forms of topological and measure-theoretical equicontinuity: relationships with discrete spectrum and sequence entropy, Ergodic Theory and Dynamical Systems 37 (2017), 1211-1237.

[GRM19] F. García-Ramos and B. Marcus, Mean sensitive, mean equicontinuous and almost periodic functions for dynamical systems, Discrete \& Continuous Dynamical Systems 39 (2019), 729-746.

[Her79] M. R. Herman, Sur la Conjugaison Différentiable des Difféomorphismes du Cercle a des Rotations, Publications Mathématiques de l'Institut des Hautes Études Scientifiques 49 (1979), 5-233.

[HJ97] K. N. Haddad and A. S. A. Johnson, Auslander systems, Proceedings of the American Mathematical Society 125 (1997), 2161-2170.

$\left[\mathrm{HLT}^{+} 21\right]$ W. Huang, J. Li, J. Thouvenot, L. Xu and X. Ye, Bounded complexity, mean equicontinuity and discrete spectrum, Ergodic Theory and Dynamical Systems 41 (2021), 494-533.

[Hos86] B. Host, Valeurs propres des systèmes dynamiques définis par des substitutions de longueur variable, Ergodic Theory and Dynamical Systems 6 (1986), 529-540.

[Hua79] T. J. Huang, A characterization of maximally almost periodic groups, Proceedings of the American Mathematical Society 75 (1979), 59-62.

[Ků03] P. Kůrka, Topological and Symbolic Dynamics, Cours Spécialisés, Vol. 11, Société mathématique de France, Paris, 2003.

[KLS15] J. Kellendonk, D. Lenz and J. Savinien (eds.), Mathematics of Aperiodic Order, Progress in Mathematics, Vol. 309, Birkhäuser/Springer, Basel, 2015.

[Len09] D. Lenz, Continuity of eigenfunctions of uniquely ergodic dynamical systems and intensity of Bragg peaks, Communications in Mathematical Physics 287 (2009), 225-258.

[Len19] D. Lenz, An autocorrelation and discrete spectrum for dynamical systems on metric spaces, Ergodic Theory and Dynamical Systems 41 (2021), 906-922.

[Lin01] E. Lindenstrauss, Pointwise theorems for amenable groups, Inventiones Mathematicae 146 (2001), 259-295. 
[LR68] H. Leptin and L. Robertson, Every locally compact map group is unimodular, Proceedings of the American Mathematical Society 19 (1968), 1079-1082.

[LS18] M. Łącka and M. Straszak, Quasi-uniform convergence in dynamical systems generated by an amenable group action, Journal of the London Mathematical Society 98 (2018), 687-707.

[LTY15] J. Li, S. Tu and X. Ye, Mean equicontinuity and mean sensitivity, Ergodic Theory and Dynamical Systems 35 (2015), 2587-2612.

[Mac64] G. W. Mackey, Ergodic transformation groups with a pure point spectrum, Illinois Journal of Mathematics 8 (1964), 593-600.

[McS93] P. D. McSwiggen, Diffeomorphisms of the torus with wandering domains, Proceedings of the American Mathematical Society 117 (1993), 1175-1186.

[McS95] P. D. McSwiggen, Diffeomorphisms of the k-torus with wandering domains, Ergodic Theory and Dynamical Systems 15 (1995), 1189-1205.

[MR13] P. Müller and C. Richard, Ergodic properties of randomly coloured point sets, Canadian Journal of Mathematics 65 (2013), 349-402.

[NS96] A. Norton and D. Sullivan, Wandering domains and invariant conformal structures for mappings of the 2-torus, Annales Academiae Scientiarum Fennicae. Series A I. Mathematica 21 (1996), 51-68.

[NV94] A. Norton and J. A. Velling, Conformal irregularity for Denjoy diffeomorphisms of the 2-torus, Rocky Mountain Journal of Mathematics 24 (1994), 655-671.

[Oxt52] J. C. Oxtoby, Ergodic sets, Bulletin of the American Mathematical Society 58 (1952), 116-136.

[Que10] M. Queffélec, Substitution Dynamical Systems-Spectral Analysis, Lecture Notes in Mathematics, 1294, Springer, Berlin, 2010.

[QZ20] J. Qiu and J. Zhao, A note on mean equicontinuity, Journal of Dynamics and Differential Equations 32 (2020), 101-116.

[Rob96] E. A. Robinson, The dynamical properties of Penrose tilings, Transactions of the American Mathematical Society 348 (1996), 4447-4464.

[Rob99] E. A. Robinson, Jr., On the table and the chair, Indagationes Mathematicae 10 (1999), 581-599.

[Sch99] M. Schlottmann, Generalized model sets and dynamical systems, in Directions in Mathematical Quasicrystals, CRM Monograph Series, Vol. 13, American Mathematical Society, Providence, RI, 2000, pp. 143-159.

[Ser85] C. Series, The geometry of Markoff numbers, The Mathematical Intelligencer 7 (1985), 20-29.

[Sie18] W. Sierpiński, Un théorème sur les continus, Tohoku Mathematical Journal 13 (1918), 300-303.

[Sol07] B. Solomyak, Eigenfunctions for substitution tiling systems, in Probability and Number Theory-Kanazawa 2005, Advanced Studies in Pure Mathematics, Vol. 49, Mathematical Society of Japan, Tokyo, 2007, pp. 433-454.

[Vee65] W. A. Veech, Almost automorphic functions on groups, American Journal of Mathematics 87 (1965), 719-751.

[vN34] J. von Neumann, Almost periodic functions in a group, Transactions of the American Mathematical Society 36 (1934), 445-492. 
[Vor12] Y. Vorobets, Notes on the Schreier graphs of the Grigorchuk group, in Dynamical Systems and Group Actions, Contemporary Mathematics, Vol. 567, American Mathematical Society, Providence, RI, 2012, pp. 221-248.

[Wal82] P. Walters, An Introduction To Ergodic Theory, Graduate Texts in Mathematics, Vol. 79, Springer, Berlin, 1982.

[YZZ19] T. Yu, G. Zhang and R. Zhang, Discrete spectrum for amenable group actions, Discrete and Continuous Dynamical Systems 41 (2021), 5871-5886. 